Supporting Information for:

\title{
Protease FRET reporters targeting neutrophil extracellular traps
}

Matteo Guerra ${ }^{1,2,3, \neq}$, Victoria S Halls ${ }^{4, \neq}$, Jolanthe Schatterny ${ }^{3}$, Matthias Hagner ${ }^{3}$, Marcus A Mall $1,3,5,6,7, *$, Carsten Schultz ${ }^{1,3,4, *, \#}$

${ }^{1}$ Molecular Medicine Partnership Unit (MMPU), European Molecular Biology Laboratory (EMBL) and University of Heidelberg, 69117 Heidelberg, Germany

${ }^{2}$ Faculty of Biosciences, Collaboration for Joint Ph.D. Degree between EMBL and Heidelberg University, 69117 Heidelberg, Germany

${ }^{3}$ Translational Lung Research Center Heidelberg (TLRC), German Center for Lung Research (DZL), 69120 Heidelberg, Germany

${ }^{4}$ Dept. of Chemical Physiology and Biochemistry, Oregon Health and Science University, Portland, OR, 97239, USA

${ }^{5}$ Dept. of Pediatric Pulmonology, Immunology and Critical Care Medicine, Charité Universitätsmedizin Berlin, 13353 Berlin, Germany ${ }^{6}$ Berlin Institute of Health, 10178 Berlin, Germany

${ }^{7}$ German Center for Lung Research (DZL), associated partner site, 13353 Berlin, Germany 


\section{Contents}

Materials

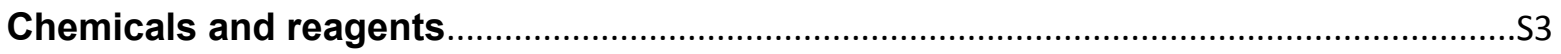

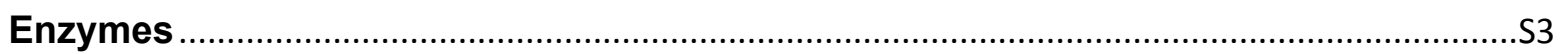

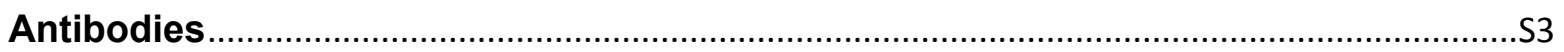

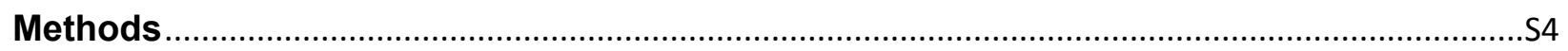

Synthetic chemistry and compounds characterization .....................................................S4

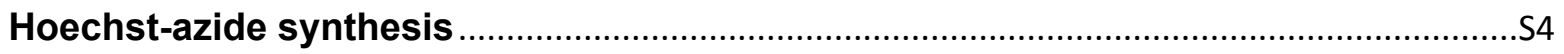

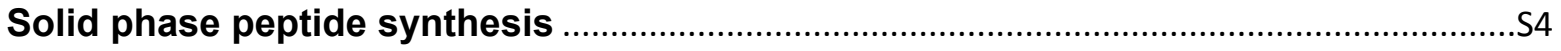

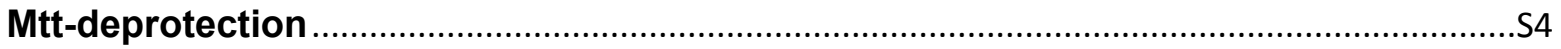

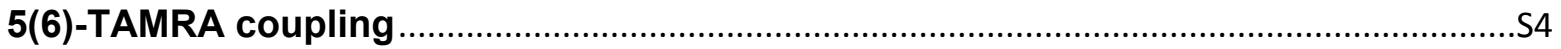

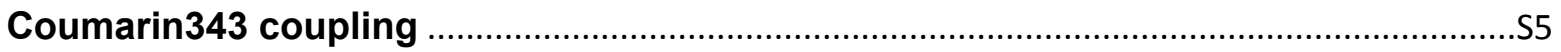

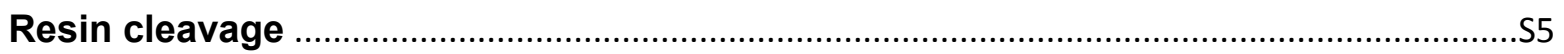

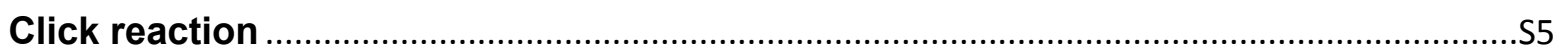

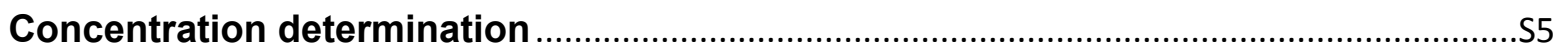

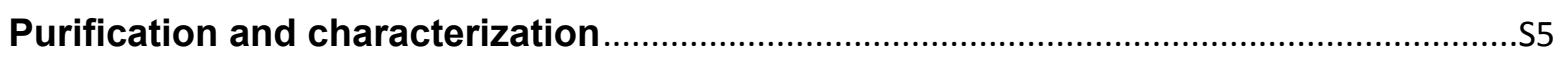

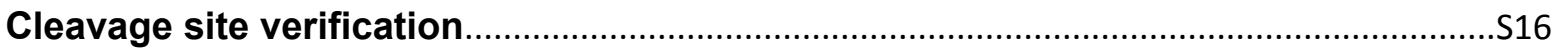

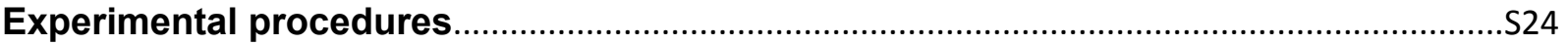

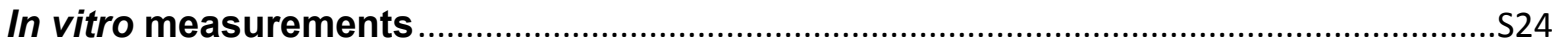

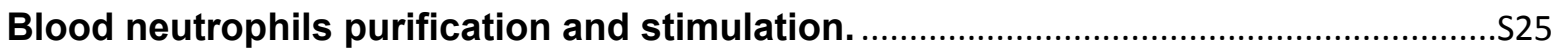

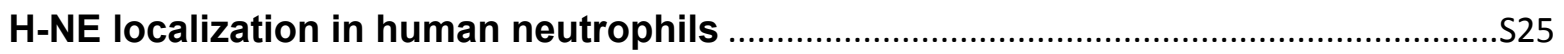

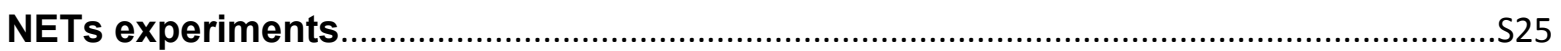

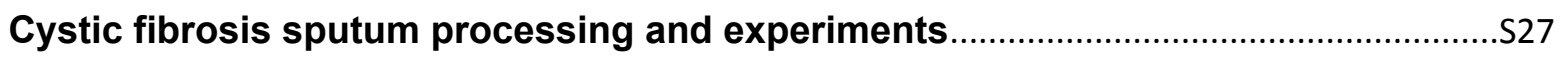

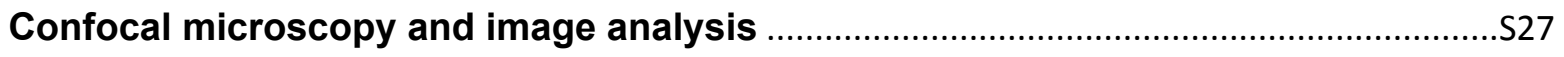

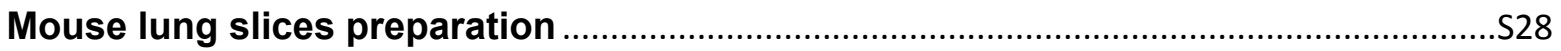

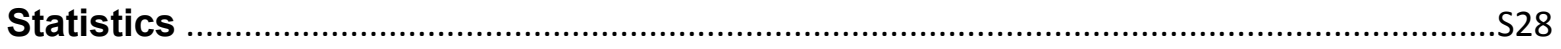

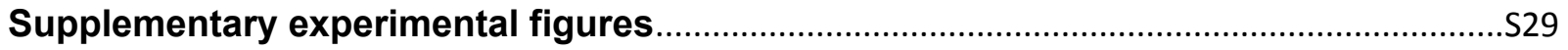

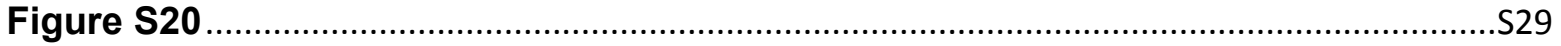

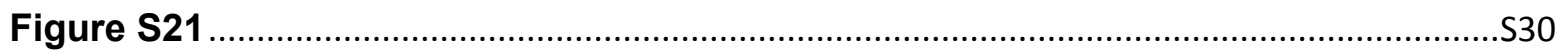

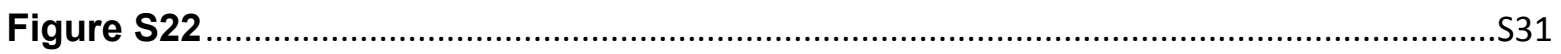

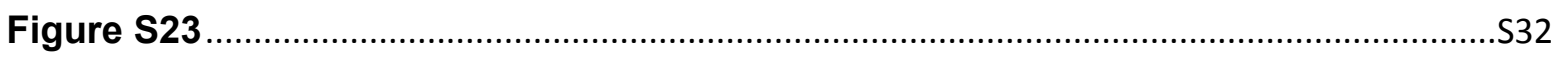

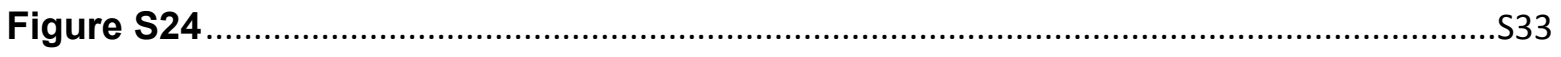

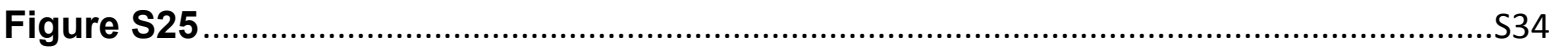


Figure S26. S35

Ethical Statement and probes availability.

Significant Hazards or Risks Statement. S37

References S36

\section{Materials}

\section{Chemicals and reagents}

All standard solvents and trifluoroacetic acid were purchased from Fisher Scientific. Standard Fmoc-protected amino acids and preloaded Wang resin were purchased from Novabiochem (Millipore Sigma). Fmoc-propargylglycine was from Matrix Scientific and [2-[2-(Fmocamino)ethoxy]ethoxy]acetic acid (peg linker) was purchased from Iris Biotech $\mathrm{GmbH}$. Coumarin343 was purchased from Acros Organics and 5(6)-TAMRA was from AnaSpec, Inc. 4Dimethylaminopyridine (DMAP), Triisopropyl silane (TIS), Diisopropylethylamine (DIPEA), Tetrakis(acetonitrile)copper(I) tetrafluoroborate, Tris[(1-benzyl-1H-1,2,3-triazol-4-yl)methyl]amine (TBTA) and N,N'-Diisopropylcarbodiimide (DIC) were purchased from TCl America. 1-(3Dimethylaminopropyl)-3-ethylcarbodiimide hydrochloride (EDCI) and (1-cyano-2-ethoxy-2oxoethylidenaminooxy)dimethylamino-morpholino-carbenium hexafluorophosphate (COMU) were purchased from Alfa Aesar. Oxyma was purchased from CEM Corporation and BromoPEG2-azide was from BroadPharm. Piperidine and bisBenzimide $\mathrm{H} 33258$ were purchased from Sigma-Aldrich. Hoechst 33342 (Cat. No.: 62249) was purchased form Thermo Fisher Scientific. Tris Buffered Saline (Cat. No.: T5030-50TAB) was form Sigma Aldrich. BlockAid ${ }^{\text {TM }}$ Blocking Solution (Cat. No.: B10710) was from Thermo Fisher Scientific ， Phorbol 12-myristate 13acetate (Cat. No.: $\quad$ P8139) was from Sigma Aldrich (Merck). DRAQ5 ${ }^{\mathrm{TM}}$ (Cat. No.: 62251) was from Thermo Fisher Scientific. Cathepsin G Inhibitor I - CAS 429676-93-7 - (Cat. No.: 219372) was from Calbiochem (Merck Millipore). Sivelestat (Cat. No.: 3535) was purchased from Tocris Bioscience.

\section{Enzymes}

Human cathepsin G (Cat. No.: SG623, purity > 95\%. Specific activity: 2 - 4 units per mg of powder), proteinase 3 (Cat. No.: ML734, purity $>95 \%$. Specific activity: $>600$ units per mg of protein) and neutrophil elastase (Cat. No.: SE563, purity > 95\%. Specific activity: 15,000-19,000 units per mg of protein) are purified of human sputum and were purchased from Elastin Products Company, Inc. Recombinant cathepsin S (Cat. No.: 1183-CY-010, purity $>95 \%$. Specific activity: $>300 \mathrm{pmol} / \mathrm{min} / \mu \mathrm{g}$, produced in and purified from the NS0 Mouse myeloma cell line) and MMP12 (917-MPB-020, purity $>95 \%$. Specific activity: $>500 \mathrm{pmol} / \mathrm{min} / \mu \mathrm{g}$, produced in and purified from the NS0 Mouse myeloma cell line) were from R\&D Systems. Human chymase (Cat. No.: BMLSE281-0010, purity $\geq 95 \%$. Specific activity: 1155 units per $\mathrm{mg}$ of protein, purified form human skin) was from Enzo Life Sciences, Inc. Mouse Recombinant Cathepsin G (CTSG) (Cat. No.: RPD993Mu01, purity: $>90 \%$ ) and mouse Recombinant Proteinase 3 (PR3) (Cat. No.: RPB434Mu01, purity: >90\%) were of prokaryotic expression (E. coli) and purchased from Cloud Clone. Recombinant Mouse Neutrophil Elastase/ELA2 Protein (Cat. No.: 4517-SE-010, purity > 95\%, Specific activity: $500 \mathrm{pmol} / \mathrm{min} / \mu \mathrm{g}$, produced and purified from the NSO Mouse myeloma cell 
line) and mouse active Cathepsin C/DPPI (rmCathepsin C) (Cat. No.: 2336-CY) were from R\&D Systems. Zinc metalloproteinase aureolysin recombinant Protein (Cat. No.: MBS1172919, purity $>90 \%$, produced in and purified from E. coli) was form MyBioSource.

\section{Antibodies}

The mouse primary antibody anti human ELA2 (neutrophil elastase) (Cat. No.: MAB91671, clone number: 950317) was from R\&D systems and the rabbit primary antibody anti human cathepsin G (Cat. No.: 703590, clone number: 12H15L69) was form Invitrogen. The anti-mouse secondary antibody (anti-mouse Ig1, Cat. No.: A21240, conjugated with Alexa Fluor 647) was form Invitrogen and the anti-rabbit secondary antibody (anti-rabbit IgG, Cat. No.: A21245, conjugated with Alexa Fluor 647) was from Thermo Fisher Scientific.

\section{Methods}

\section{Synthetic chemistry and compounds characterization}

\section{Hoechst-azide synthesis}

In order to obtain free bisBenzimide $\mathrm{H} 33258$, the hydrochloric acid salt (100 $\mathrm{mg}, 0.187 \mathrm{mmol}$ ) was dissolved in water $(1.87 \mathrm{~mL})$. Potassium carbonate $(1.8 \mathrm{~g}, 13 \mathrm{mmol})$ was added and the suspension was vortexed and centrifuged in an Eppendorf $5430 \mathrm{R}$ at $4000 \mathrm{rpm}$ for $5 \mathrm{~min}$. The solvent was removed and the precipitate washed with water $(4 \mathrm{~mL})$. The suspension was vortexed and centrifuged at $7840 \mathrm{rpm}$ for $15 \mathrm{~min}$. The supernatant was then discarded and the precipitate freeze dried for 48 hours.

The so obtained free bisBenzimide $\mathrm{H} 33258$ (105 $\mathrm{mg}, 0.247 \mathrm{mmol}$ ) was dissolved in dimethylformamide $(1.3 \mathrm{~mL})$ and bromo-Peg2-azide $(77 \mathrm{mg}, 0.322 \mathrm{mmol})$ and potassium carbonate $(93 \mathrm{mg}, 0.667 \mathrm{mmol})$ were added. The solution was shaken for 18 hours at $1000 \mathrm{rpm}$ and $60^{\circ} \mathrm{C}$ on an Eppendorf ThermoMixer $\mathrm{C}$. The solvent was then evaporated under high vacuum at $40{ }^{\circ} \mathrm{C}$ and the residue was dissolved in acetonitrile/water $(1: 1,8 \mathrm{~mL})$ containing formic acid $(0.1 \% \mathrm{v} / \mathrm{v})$ for HPLC purification (Figure S1). Purified Hoechst-azide (15 mg, $25.8 \mu \mathrm{mol})$ was lyophilized and dissolved in dimethyl sulfoxide to a concentration of $40 \mathrm{mg} / \mathrm{mL}$. Hoechst azide: ${ }^{1} \mathrm{H}$ NMR (400 MHz, MeOD) $\delta 8.23(\mathrm{~d}, J=1.0 \mathrm{~Hz}, 1 \mathrm{H}), 8.03(\mathrm{~d}, J=8.9 \mathrm{~Hz}, 2 \mathrm{H}), 7.93$ (dd, $J=8.5,1.6$ $\mathrm{Hz}, 1 \mathrm{H}), 7.67(\mathrm{~d}, J=8.5 \mathrm{~Hz}, 1 \mathrm{H}), 7.51(\mathrm{~d}, J=8.8 \mathrm{~Hz}, 1 \mathrm{H}), 7.14(\mathrm{~d}, J=2.0 \mathrm{~Hz}, 1 \mathrm{H}), 7.08(\mathrm{~d}, J=$ $8.9 \mathrm{~Hz}, 2 \mathrm{H}), 7.04(\mathrm{dd}, J=8.8,2.2 \mathrm{~Hz}, 1 \mathrm{H}), 4.23-4.13(\mathrm{~m}, 2 \mathrm{H}), 3.86(\mathrm{dd}, J=5.3,3.8 \mathrm{~Hz}, 2 \mathrm{H})$, $3.74-3.70(\mathrm{~m}, 2 \mathrm{H}), 3.70-3.65(\mathrm{~m}, 4 \mathrm{H}), 3.39-3.34(\mathrm{~m}, 2 \mathrm{H}), 3.30-3.25(\mathrm{~m}, 4 \mathrm{H}), 2.97-2.84$ (m, 4H), 2.55 (s, 3H) (Figure S3).

\section{Solid phase peptide synthesis}

Peptides were synthesized on a CEM Liberty Blue Automated Microwave Peptide Synthesizer, using an $0.1 \mathrm{mmol}$ scale customized standard method. This included microwave-assisted deprotection and coupling reactions. Deprotection was carried out with $20 \%$ piperidine in dimethylformamide at $90{ }^{\circ} \mathrm{C}$ for $1: 25$ min followed by three washes with dimethylformamide. Coupling reactions were carried out with DIC $(1 \mathrm{M}, 1 \mathrm{~mL})$, Oxyma $(1 \mathrm{M}, 0.5 \mathrm{~mL})$ and the respective amino acids $(0.2 \mathrm{M}, 2.5 \mathrm{~mL})$ at $90{ }^{\circ} \mathrm{C}$ for 2:05 min and double couplings $(2 \times 2: 05 \mathrm{~min})$ for Fmocarginine. Fmoc-propargylglycine and the peg linker were used in lower concentrations $(0.08 \mathrm{M}$, $2.5 \mathrm{~mL}$ ) and therefore coupling time was extended to $10 \mathrm{~min}$. Deprotection of the final $\mathrm{N}$-terminal amino acid was not carried out at this stage of synthesis. 


\section{Mtt-deprotection}

The selective deprotection of 4-methyltrityl-protected lysine was carried out manually by adding $2 \%$ trifluoroacetic acid in dichloromethane $(4 \mathrm{~mL})$ to the resin and shaking it at $700 \mathrm{rpm}$ on an Eppendorf ThermoMixer $\mathrm{C}$ at room temperature for $4 \mathrm{~min}$ and subsequently washing it three times with dichloromethane $(4 \mathrm{~mL}$ each). This was repeated 10 times to assure total removal of the protecting group.

\section{5(6)-TAMRA coupling}

A solution of 5(6)-TAMRA (1.5 eq), DMAP (3 eq), EDCl (3 eq) in dimethylformamide (4 mL) was added to the resin $(0.1 \mathrm{mmol})$ with the deprotected lysine and shaken at $700 \mathrm{rpm}$ on an Eppendorf ThermoMixer $\mathrm{C}$ at room temperature for 15 hours. The resin was successively washed with dimethylformamide, methanol/dichloromethane (1:1) and dichloromethane, each until the wash solvent remained colorless.

\section{Coumarin343 coupling}

Deprotection of the N-terminal Fmoc-propargylglycine of the TAMRA coupled resin was achieved by using the CEM Liberty Blue Automated Microwave Peptide Synthesizer. Subsequent coupling of Coumarin343 was carried out using a customized method which enabled manual addition of the 5 minutes preincubated mixture of Coumarin343 (3 eq), COMU (3 eq) and DIPEA (6 eq) and coupling at $75{ }^{\circ} \mathrm{C}$ for 20 minutes. The resin was successively washed with dimethylformamide, dichloromethane and diethyl ether three times each and then dried under argon to prepare for resin cleavage.

\section{Resin cleavage}

For the cleavage of the A-CG peptide a mixture of trifluoroacetic acid, triisopropyl silane and water (96:2:2, $1 \mathrm{~mL}$ ) was added to the resin and shaken for 2 hours at $700 \mathrm{rpm}$ and $42{ }^{\circ} \mathrm{C}$ on an Eppendorf ThermoMixer C. For the cleavage of the A-NE peptide a mixture of trifluoroacetic acid, triisopropyl silane and 1,2-ethanedithiol (96.5/1/2.5) was added to the resin and shaken for 2 hours at $700 \mathrm{rpm}$ and $42^{\circ} \mathrm{C}$ on an Eppendorf ThermoMixer C. After 2 hours, $8 \mu \mathrm{L}$ 1,2-ethanedithiol and $6.5 \mu \mathrm{L}$ bromotrimethylsilane were added and the mixture was shaken for additional $15 \mathrm{~min}$ to reduce oxidized methionine. Peptides were then precipitated in ice-cold diethyl ether $(45 \mathrm{~mL})$ and centrifuged in an Eppendorf $5430 \mathrm{R}$ centrifuge at $7860 \mathrm{rpm}$ and $4{ }^{\circ} \mathrm{C}$ for 15 minutes. The ether was decanted and the pellet washed with another portion of ice-cold diethyl ether $(45 \mathrm{~mL})$ and centrifugation and decanting were repeated. The pellet was dried for 30 minutes under reduced pressure and dissolved in acetonitrile/water $(1: 1,8 \mathrm{~mL})$ with formic acid $(0.1 \% \mathrm{v} / \mathrm{v})$ for HPLC purification (Figure S4 and Figure S6). Purified peptides were lyophilized and dissolved in dimethyl sulfoxide to a concentration of $10 \mathrm{mM}$.

\section{Click reaction}

Purified alkyne-reporter $(10 \mathrm{mM})$ was diluted to a final concentration of $1 \mathrm{mM}$ with DMSO and Hoechst-azide (40 mg/mL, 28 eq), TBTA (105 eq) and tetrakis(acetonitrile)copper(l) tetrafluoroborate $(40 \mathrm{eq})$ were added. The reaction mixture was shaken for 15 hours at $700 \mathrm{rpm}$ on an Eppendorf ThermoMixer at room temperature under an argon atmosphere. The reaction mixture was diluted 8-fold with acetonitrile/water (1:9) containing formic acid $(0.1 \% \mathrm{v} / \mathrm{v})$ for HPLC purification (Figure S8 and Figure S10). Purified peptides were lyophilized and dissolved in dimethyl sulfoxide to a concentration of $5 \mathrm{mM}$.

\section{Concentration determination}

The concentration of alkyne- and Hoechst-reporters were determined by measuring absorbance of reporter-bound coumarin343 $\left[\varepsilon=44212 \mathrm{~cm}^{-1} \mathrm{M}^{-1}\right]$ at $444 \mathrm{~nm}$ in methanol and calculating the concentration using the Lambert-Beer equation: $A=\varepsilon c l[\mathrm{~A}=$ absorbance, $\varepsilon=$ extinction 
coefficient, $\mathrm{c}=$ concentration, $\mathrm{I}=$ path length]. Hoechst-azide absorbance at $444 \mathrm{~nm}$ is neglectable.

\section{Purification and characterization}

Analytical runs and semi-preparative runs were carried out using reverse phase HPLC on an Agilent 1260 system equipped with a photodiode array detector and coupled to an Advion expression compact mass spectrometer. Analytical runs were performed on a $4 \mathrm{~mm}$ diameter C18 reverse phase column (NUCLEODUR C18 ec $5 \mu \mathrm{m}, 4 \mathrm{~mm}$ x $250 \mathrm{~mm}$, Macherey-Nagel, Düren, Germany) using a linear gradient from 10-100 \% acetonitrile in water with formic acid $(0.1$ $\% \mathrm{v} / \mathrm{v}$ ) for 15 minutes and a flow rate of $1 \mathrm{~mL} / \mathrm{min}$. Semi-preparative runs were performed on a 10 $\mathrm{mm}$ diameter C18 revers phase column (NUCLEODUR® 100-5 C18 ec, $10 \mathrm{~mm}$ ID x $250 \mathrm{~mm}$, Macherey-Nagel, Düren, Germany) using linear gradients from 10-75 \% for Hoechst-azide, 25-50 $\%$ for A-NE, 30-80 \% for A-CG, 20-60 \% for H-NE and H-CG acetonitrile in water with formic acid $(0.1 \% \mathrm{v} / \mathrm{v})$ for 25 minutes and a flow rate of $5 \mathrm{~mL} / \mathrm{min}$. Wavelengths for detection were $254 \mathrm{~nm}$, $341 \mathrm{~nm}, 440 \mathrm{~nm}$ and $551 \mathrm{~nm}$ and analyzed ions in mass spectrometry were multiple charged. HPLC and MS raw data was recorded with the Advion Mass Express software and plotted in Microsoft Excel.

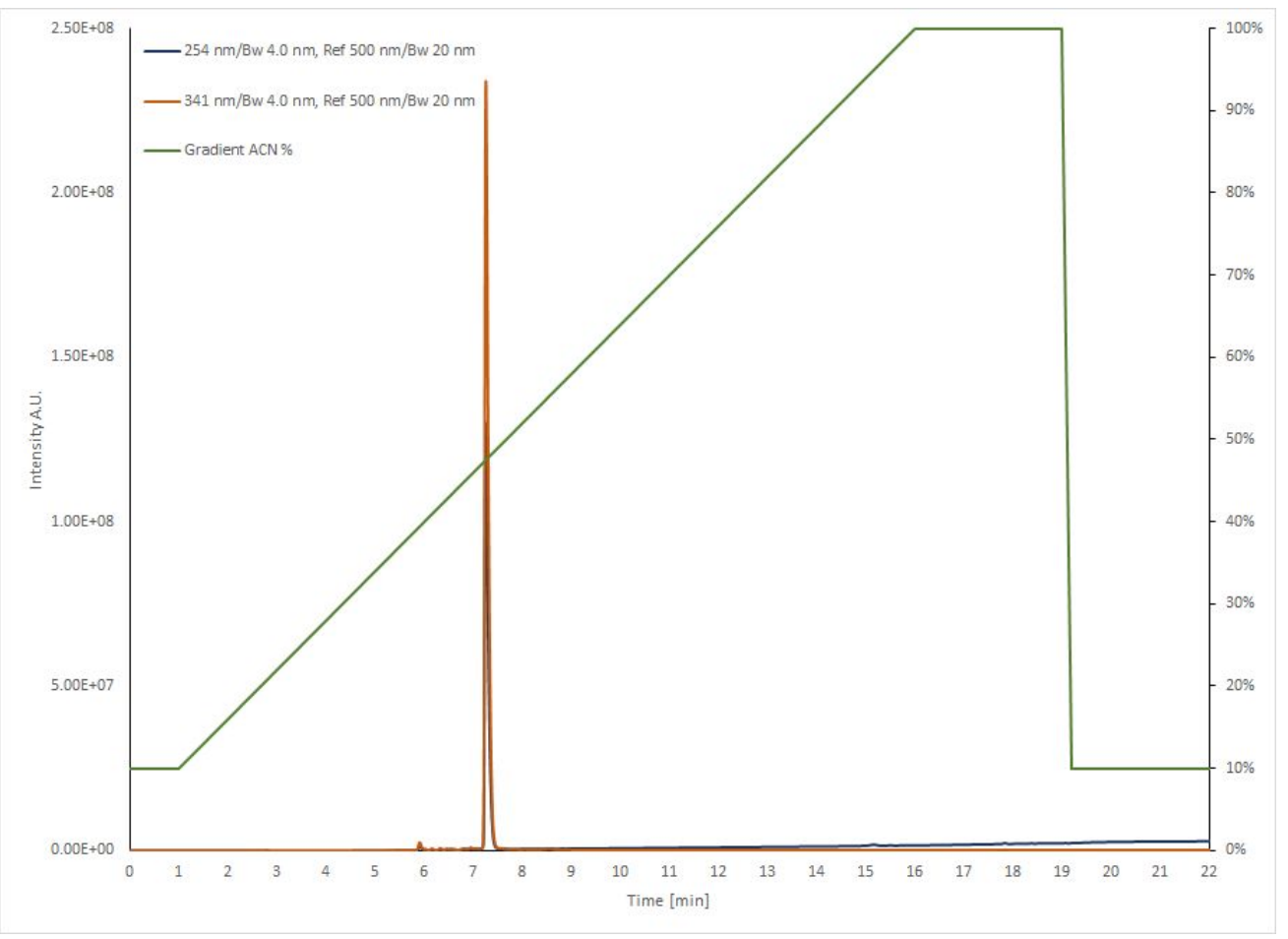

Figure S1. Analytical HPLC of purified Hoechst-azide.

Gradient $10-100 \%$ acetonitrile in water with formic acid $(0.1 \% \mathrm{v} / \mathrm{v})$. Wavelengths for detection $254 \mathrm{~nm}$ and $341 \mathrm{~nm}$ (Hoechst). 


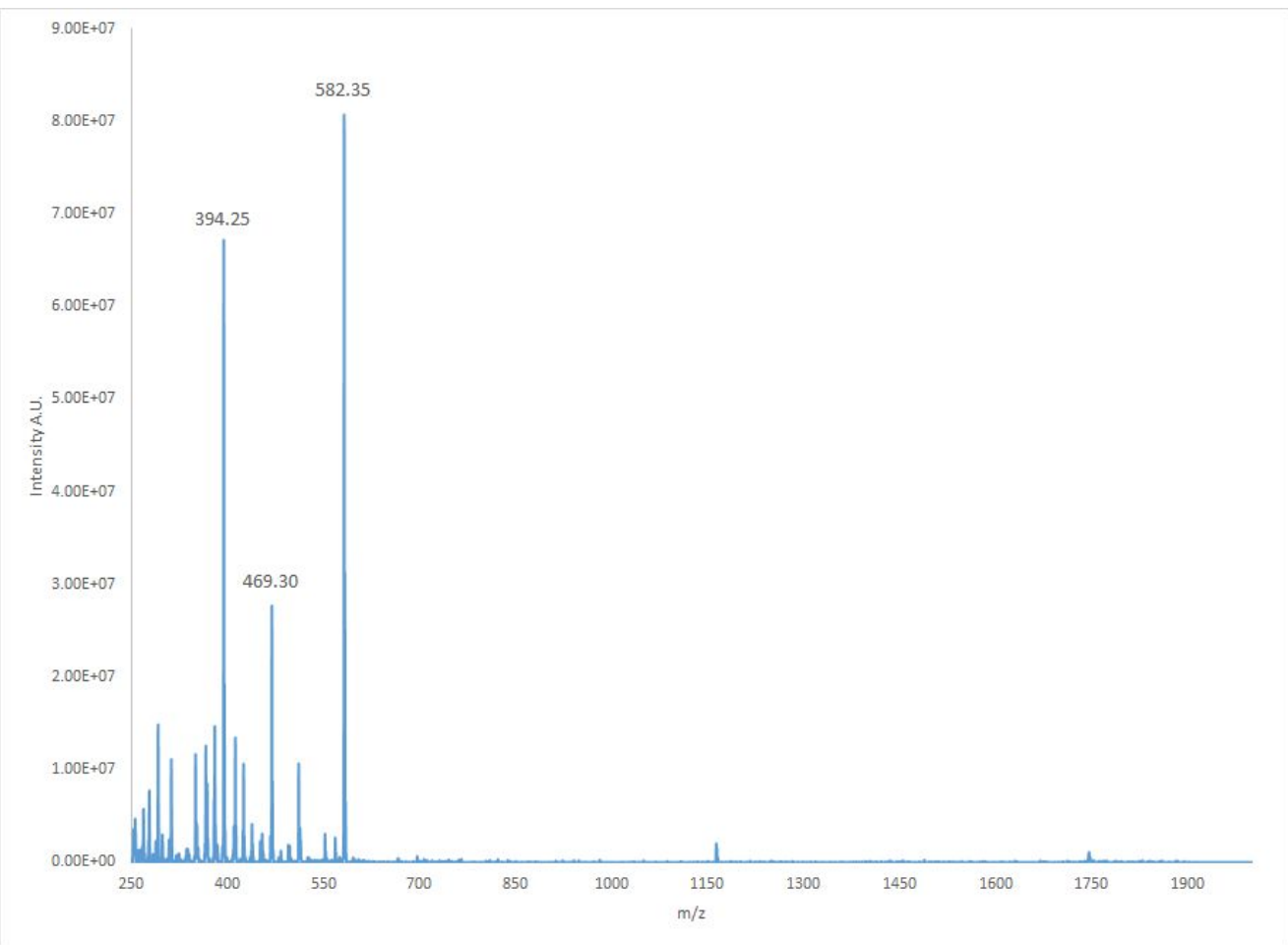

Figure S2. Mass spectrum of Hoechst-azide. Calculated mass $[M+1]^{+}=582.29$. 


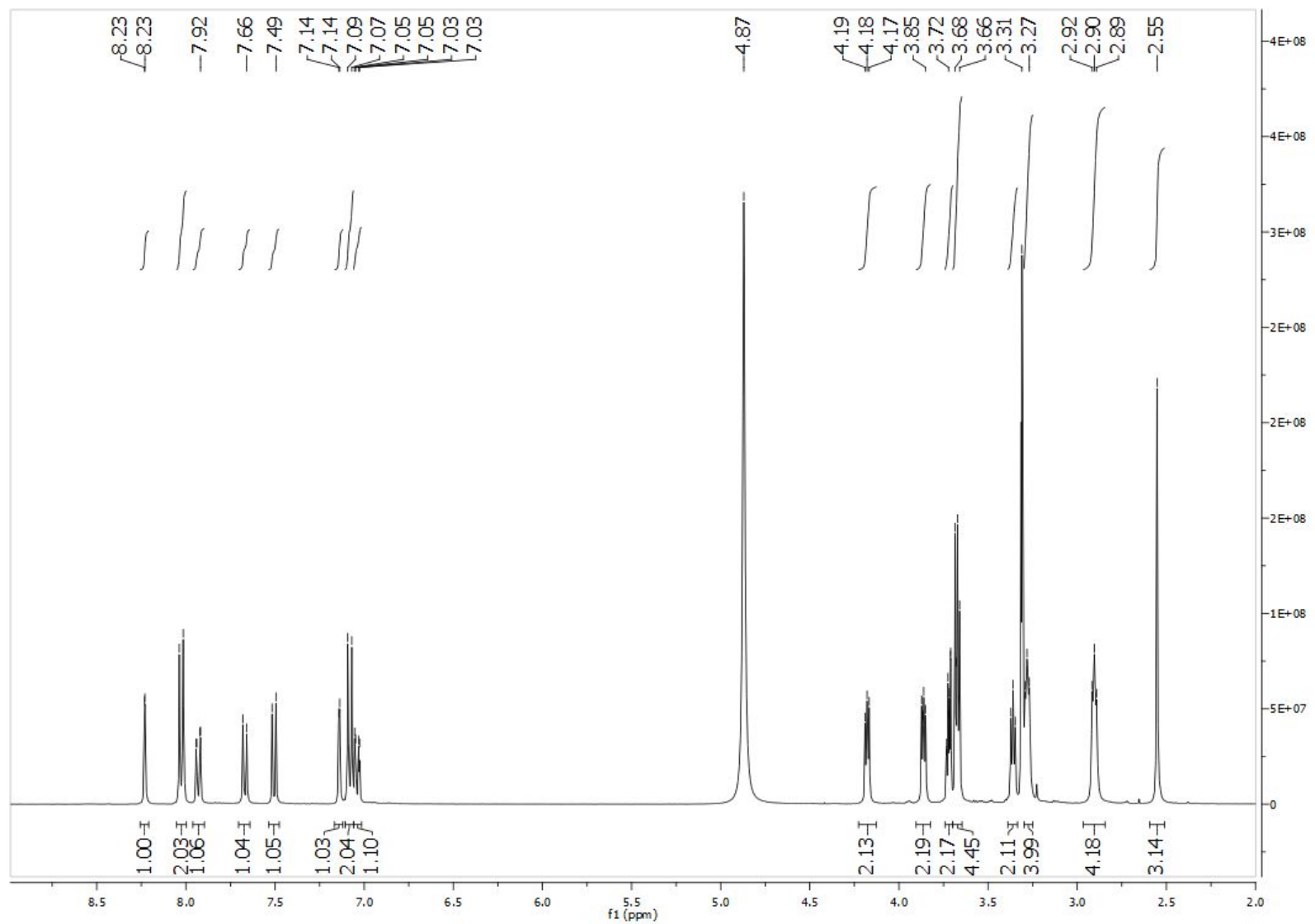

Figure S3. ${ }^{1} \mathrm{H}$-NMR of Hoechst-azide

${ }^{1} \mathrm{H}-\mathrm{NMR}$ at $400 \mathrm{MHz}$ in $\mathrm{MeOD}$. 


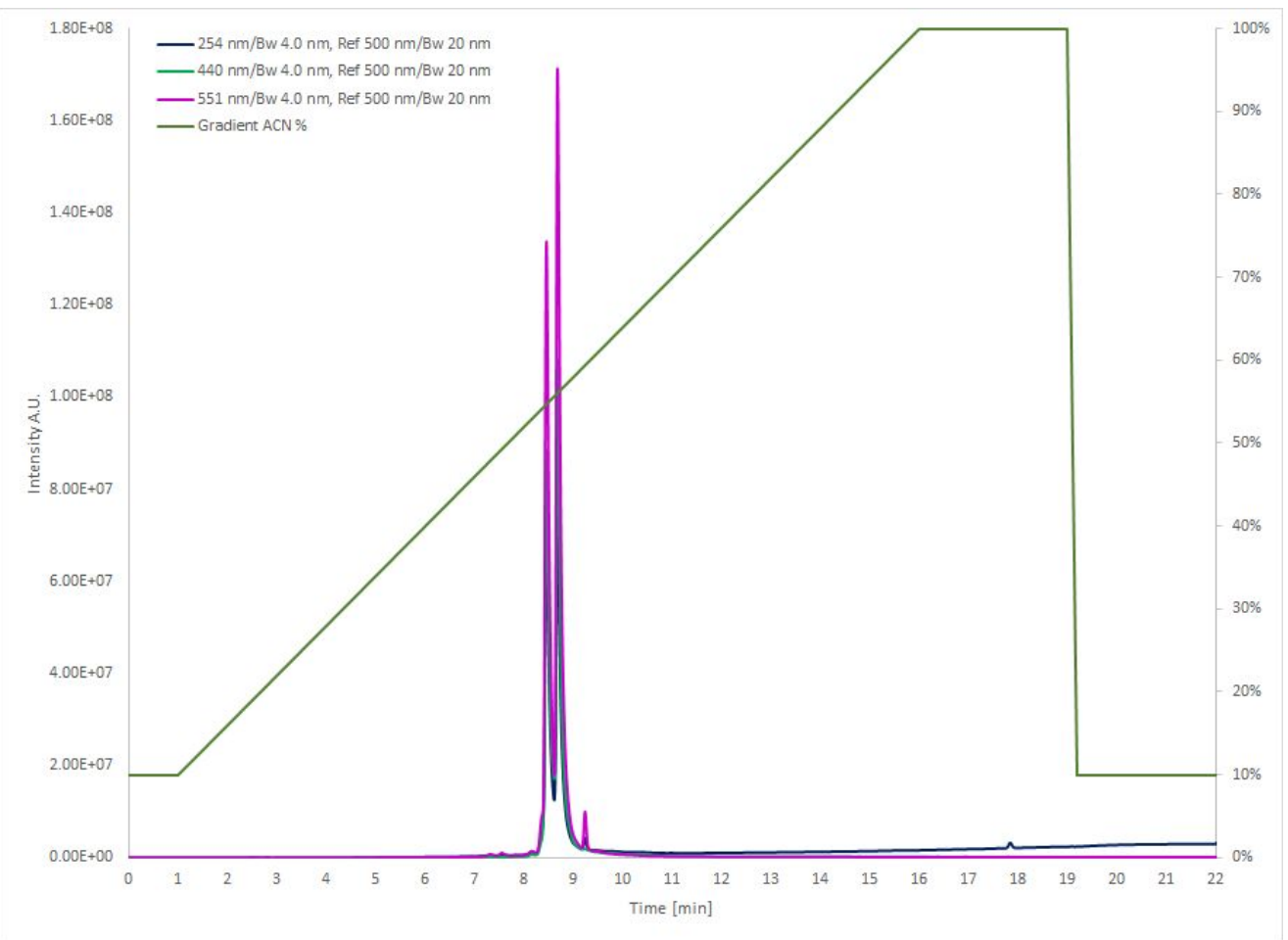

Figure S4. Analytical HPLC of purified A-NE.

Gradient $10-100 \%$ acetonitrile in water with formic acid $(0.1 \% \mathrm{v} / \mathrm{v})$. The 5(6)-TAMRA isomer mixture accounts for the double peak. Wavelengths for detection $254 \mathrm{~nm}, 440 \mathrm{~nm}$ (Coumarin343) and $551 \mathrm{~nm}$ (TAMRA). 


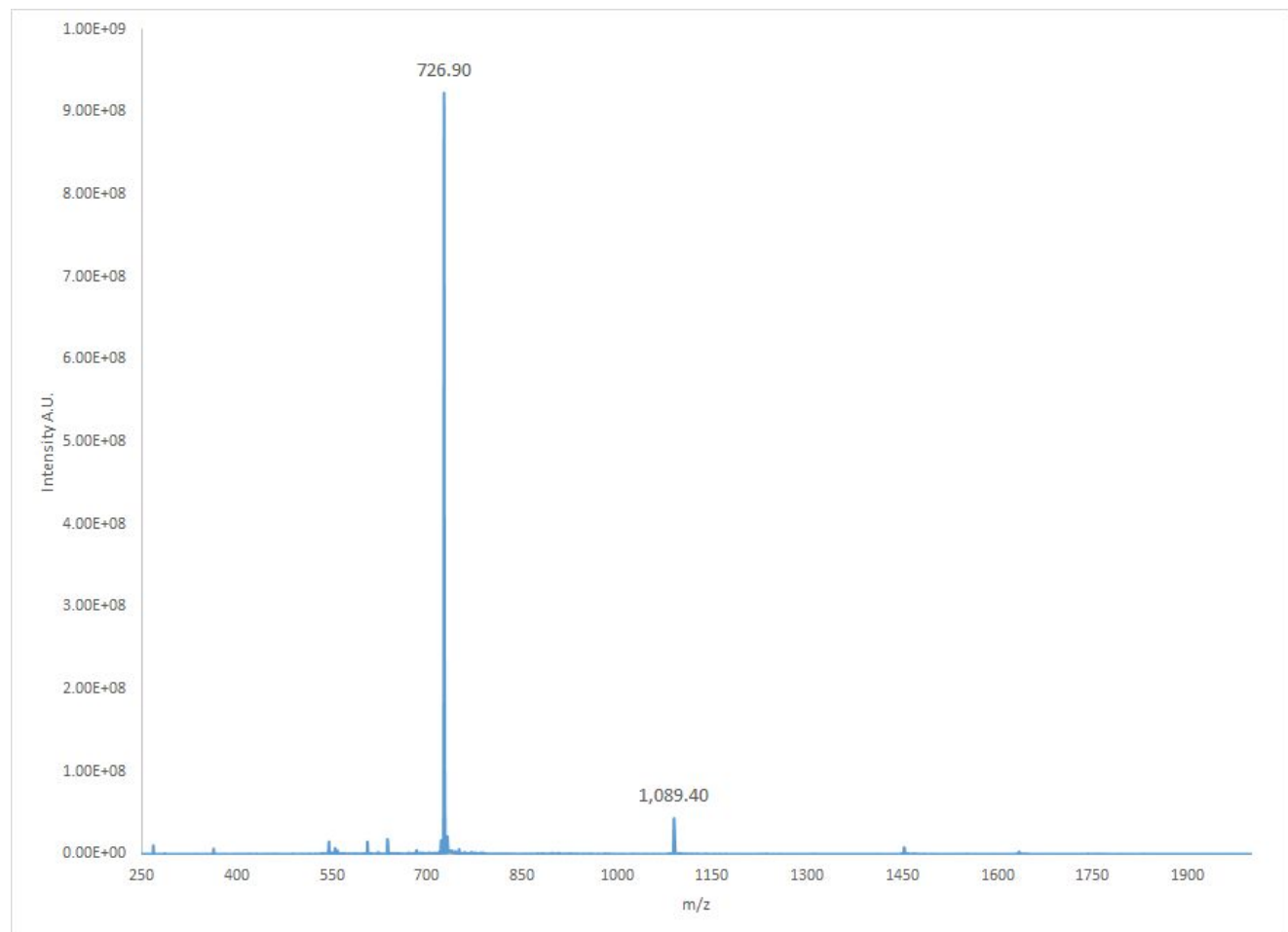

Figure S5. Mass spectrum of A-NE.

Calculated mass: $[M+1]^{+}=2177.03,[M+2]^{2+}=1089.02,[M+3]^{3+}=726.35$. 


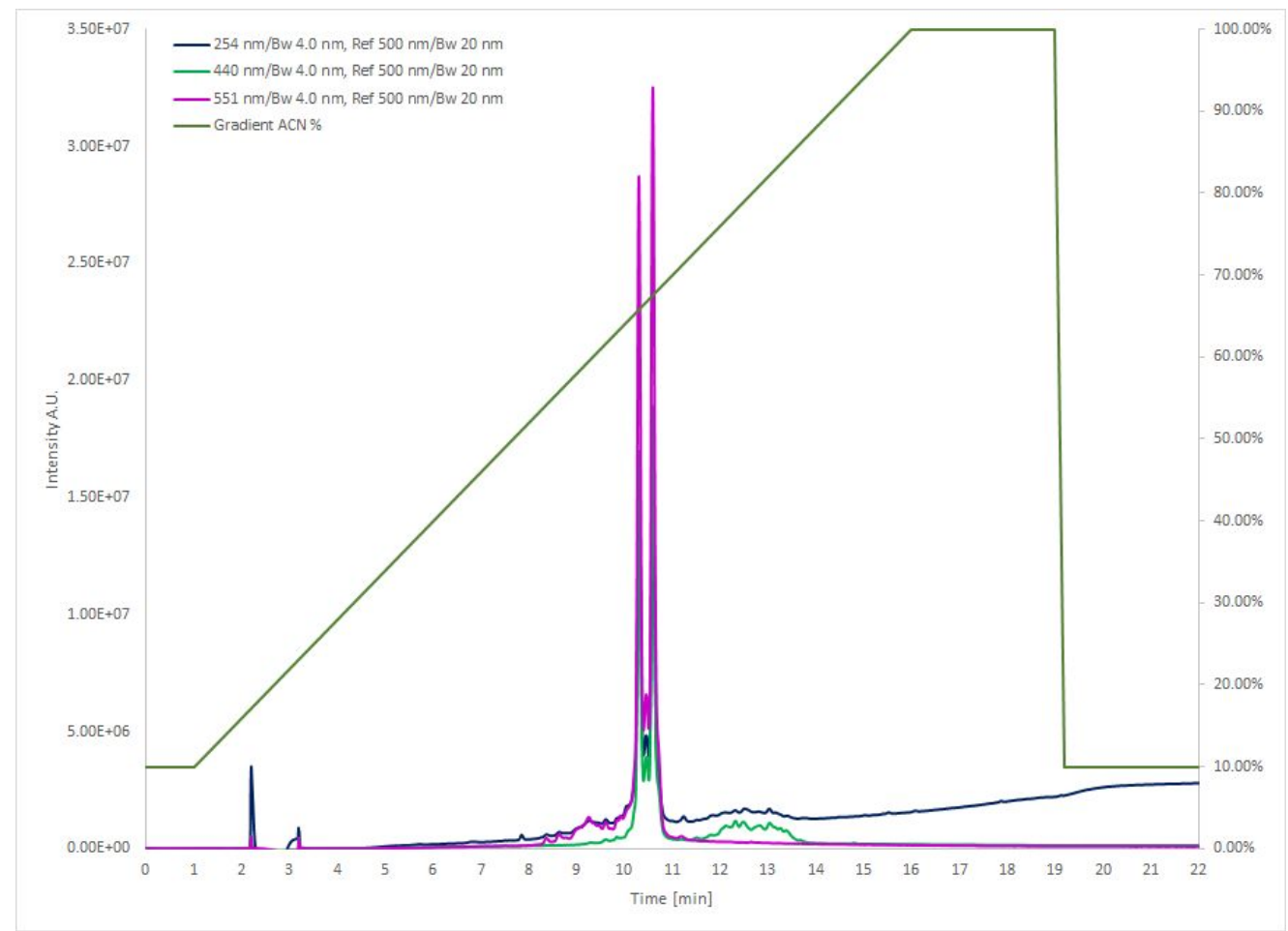

Figure S6. Analytical HPLC of purified A-CG.

Gradient $10-100 \%$ acetonitrile in water with formic acid $(0.1 \% \mathrm{v} / \mathrm{V})$. The 5(6)-TAMRA isomer accounts for the double peak. Wavelengths for detection $254 \mathrm{~nm}, 440 \mathrm{~nm}$ (Coumarin343) and $551 \mathrm{~nm}$ (TAMRA). 


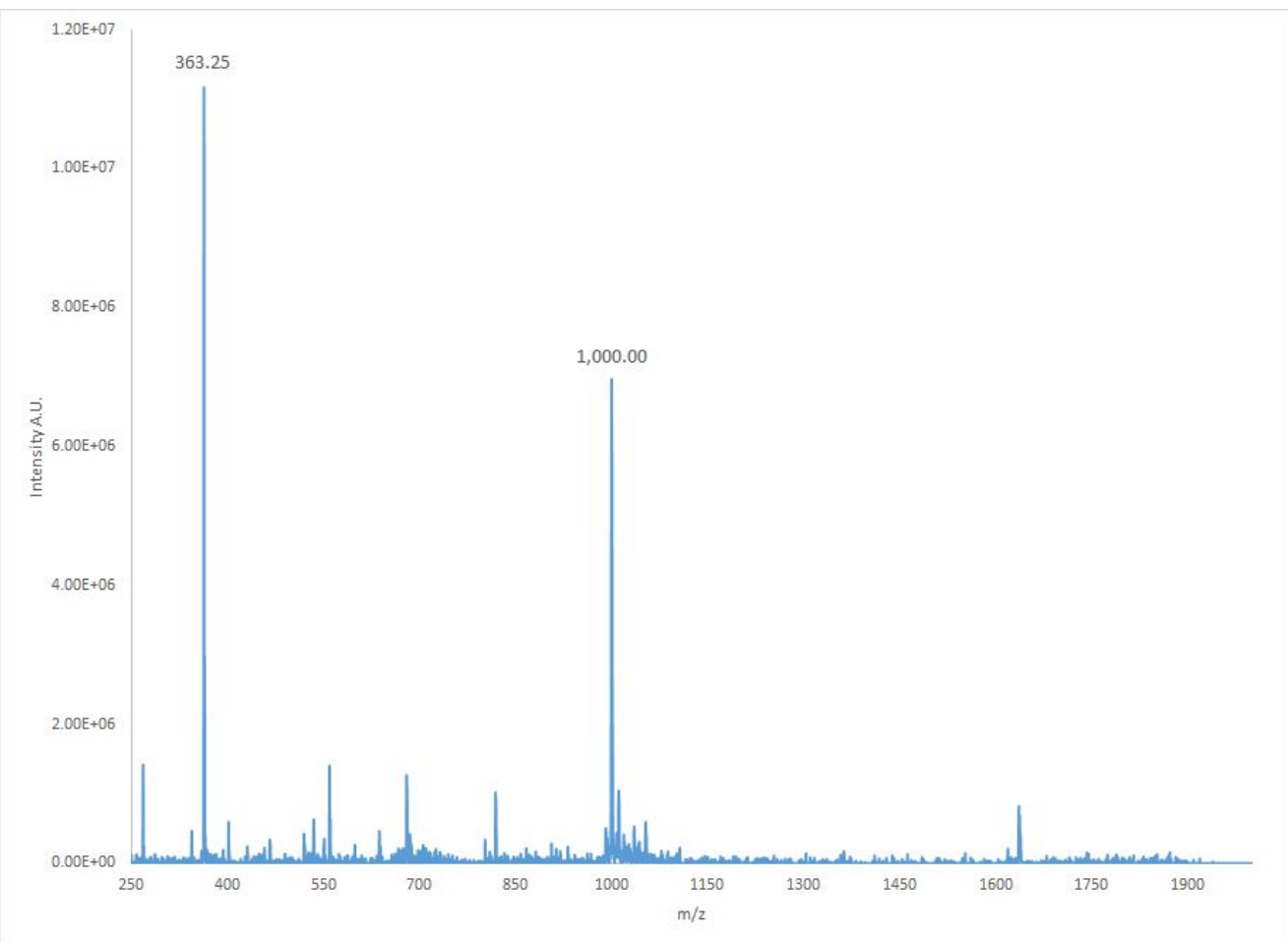

Figure S7. Mass spectrum of A-CG.

Calculated mass: $[M+1]^{+}=1997.84,[M+2]^{2+}=999.42,[M+3]^{3+}=666.62$. 


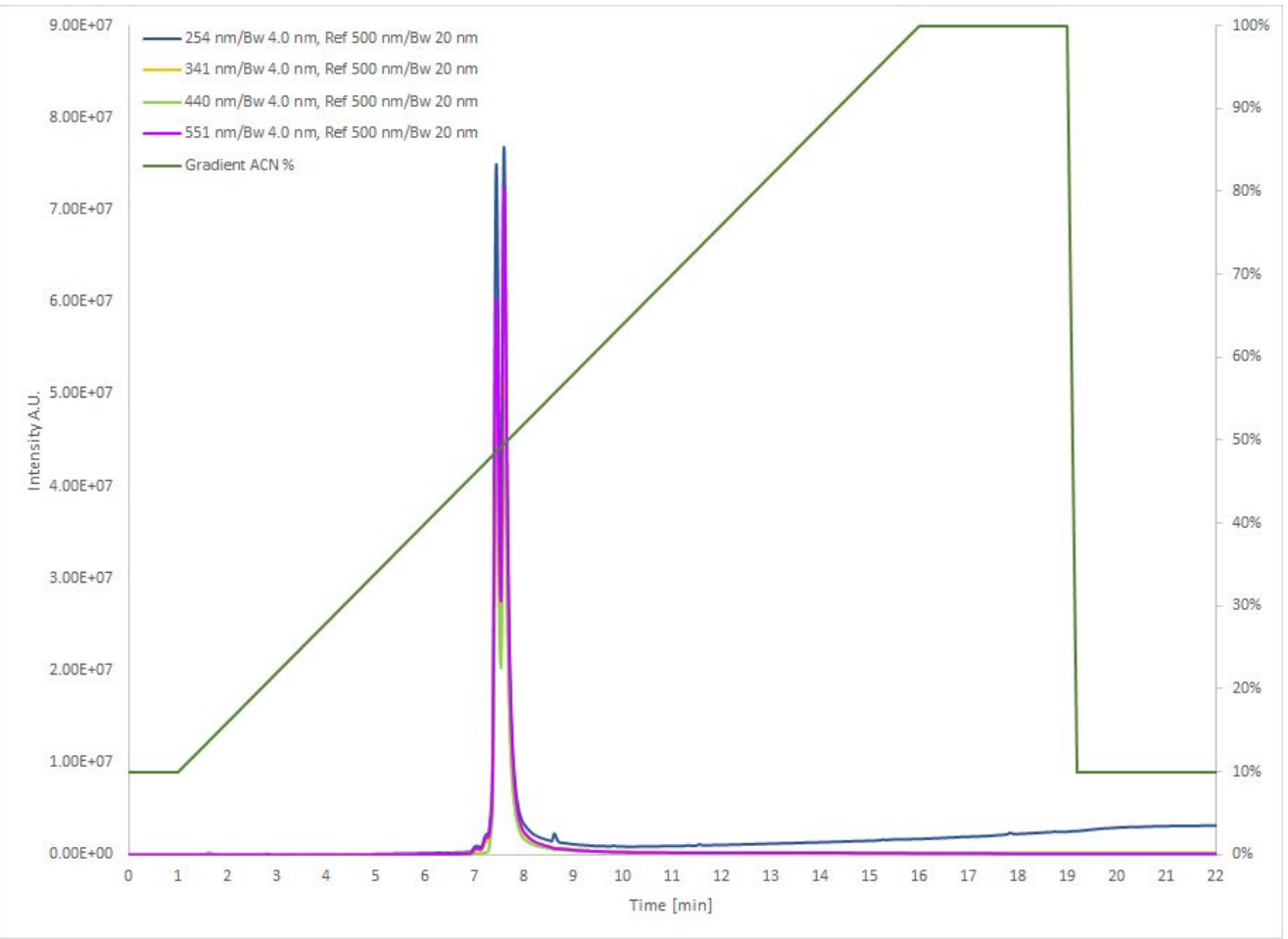

Figure S8. Analytical HPLC of purified H-NE.

Gradient $10-100 \%$ acetonitrile in water with formic acid $(0.1 \% \mathrm{v} / \mathrm{V})$. The 5(6)-TAMRA isomer accounts for the double peak. Wavelengths for detection $254 \mathrm{~nm}, 341 \mathrm{~nm}$ (Hoechst), $440 \mathrm{~nm}$ (Coumarin343) and $551 \mathrm{~nm}$ (TAMRA). 


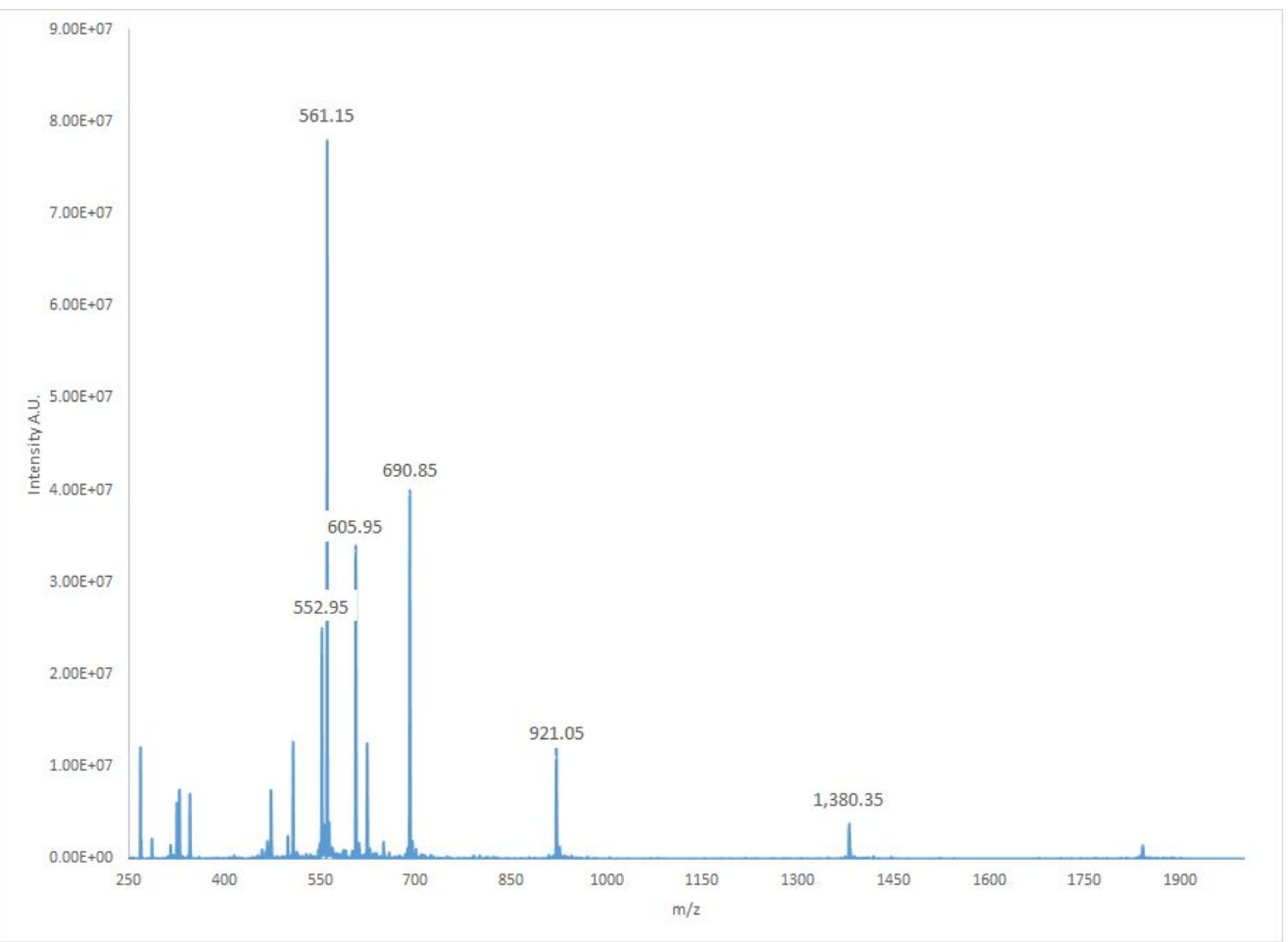

Figure S9. Mass spectrum of H-NE.

Calculated mass: $[M+1]^{+}=2758.31,[M+2]^{2+}=1379.66,[M+3]^{3+}=920.11,[M+4]^{4+}=690.33$, $[M+5]^{5+}=552.47$. 


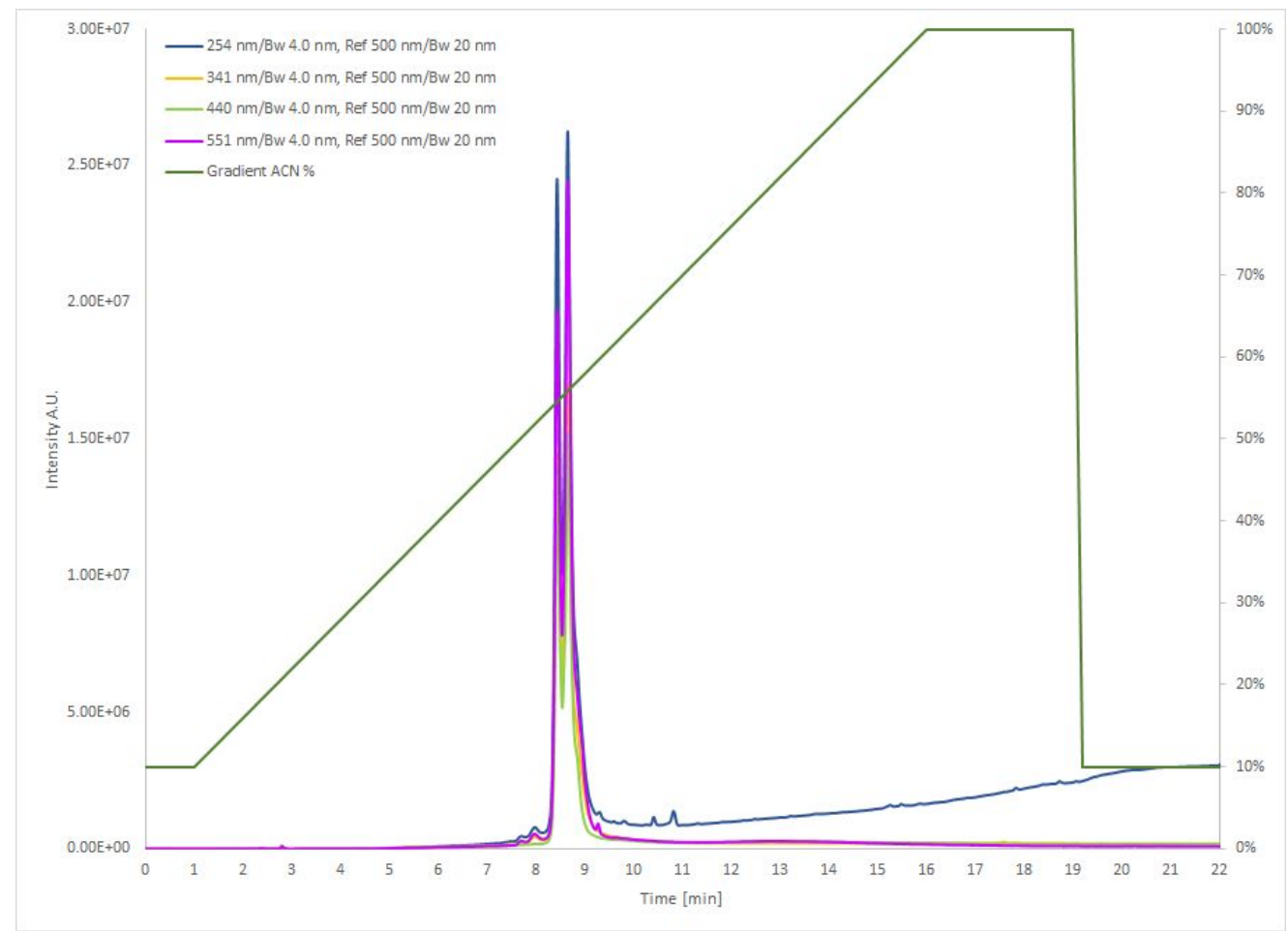

Figure S10. Analytical HPLC of purified H-CG.

Gradient $10-100 \%$ acetonitrile in water with formic acid $(0.1 \% \mathrm{v} / \mathrm{V})$. The 5(6)-TAMRA isomer accounts for the double peak. Wavelengths for detection $254 \mathrm{~nm}, 341 \mathrm{~nm}$ (Hoechst), $440 \mathrm{~nm}$ (Coumarin343) and $551 \mathrm{~nm}$ (TAMRA). 


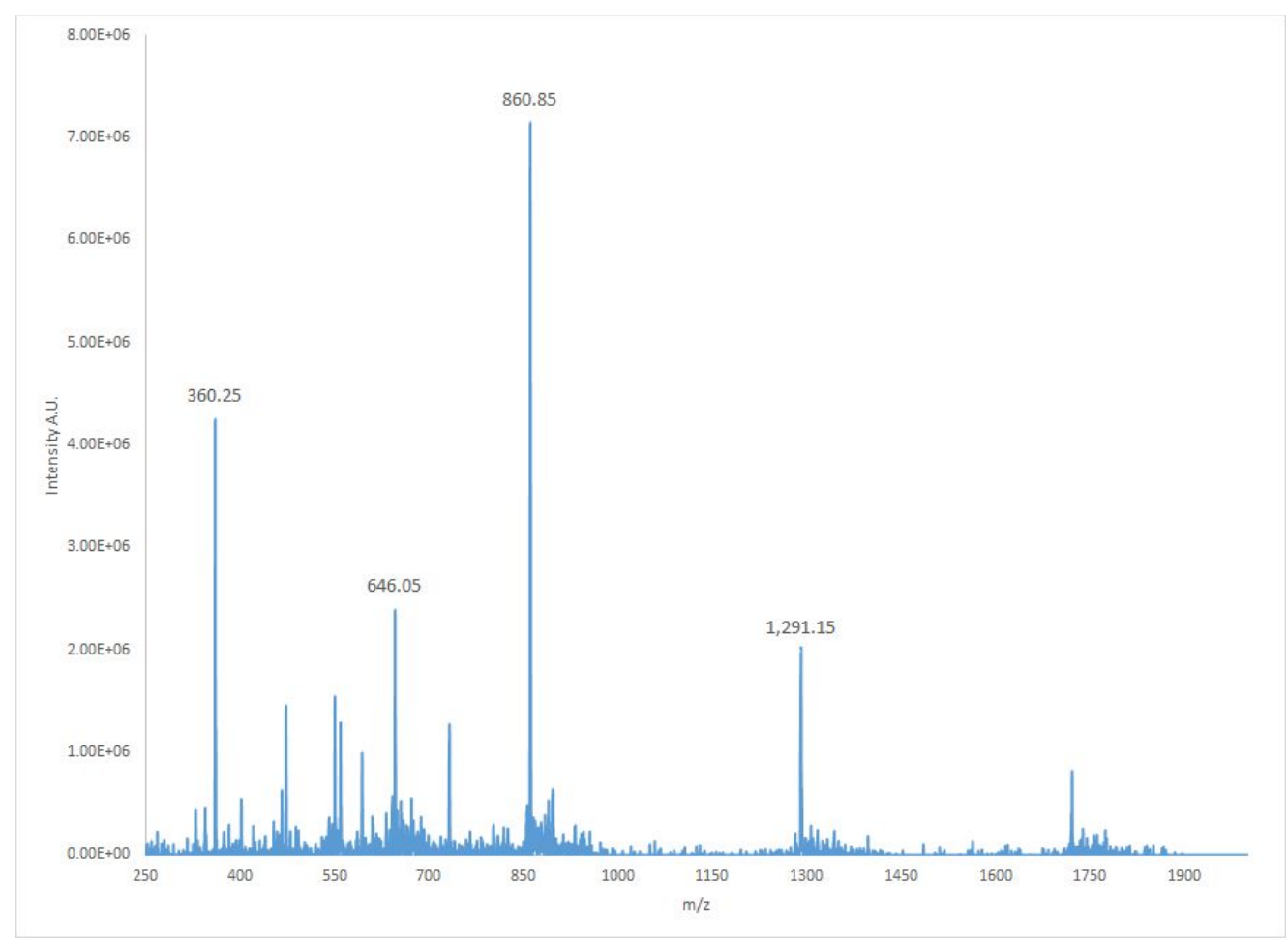

Figure S11. Mass spectrum of H-CG.

Calculated mass: $[M+1]^{+}=2579.12,[M+2]^{2+}=1290.07,[M+3]^{3+}=860.38,[M+4]^{4+}=645.54$.

\section{Cleavage site verification}

To verify the reported cleavage sites of $\mathrm{H}-\mathrm{NE}$ and $\mathrm{H}-\mathrm{CG}$, the reporters were suspended in $0.1 \mathrm{M}$ Tris- $\mathrm{HCl}, \mathrm{pH} 7.5$ to a final concentration of $1 \mathrm{mM}$ and incubated with $200 \mathrm{nM}$ enzyme for 1 hour at room temperature. The reaction was quenched by adding four volumes of absolute ethanol to denature the enzyme and then incubated for 20 minutes on ice. The sample was centrifuged at $14000 \mathrm{~g}$ for 20 minutes to separate denatured enzyme from the reaction mixture. The supernatant was collected and dried under high vacuum. The residue was dissolved in $10 \%$ acetonitrile in water with formic acid $(0.1 \% \mathrm{v} / \mathrm{v})$ and the fragments were separated and analyzed via analytical HPLC-MS.

For H-NE this resulted in two peaks with TAMRA absorbance (Peak 1 and 2, Figure S12) corresponding to the acceptor fragment, MRRQK(TAMRA) (Figure S13), two peaks with absorbance of all fluorophores (peak 3 and 4, Figure S12) corresponding to the intact reporter (Figure S14) and one peak with Hoechst and coumarin343 absorbance (peak 5, Figure S12) corresponding to the donor fragment, Coumarin343-propargylglycine(Hoechst)pegAPEEI (Figure S15). For H-CG this resulted in two peaks with TAMRA absorbance (Peak 1 and 2, Figure S16) corresponding to the acceptor fragment, WEDQK-TAMRA (Figure S17), one peak with Hoechst and coumarin343 absorbance (peak 3, Figure S16) corresponding to the donor fragment Coumarin343-propargylglycine(Hoechst)pegEPF (Figure S18) and two peaks with absorbance of all fluorophores (peak 4 and 5, Figure S16) corresponding to the intact reporter (Figure S19). 


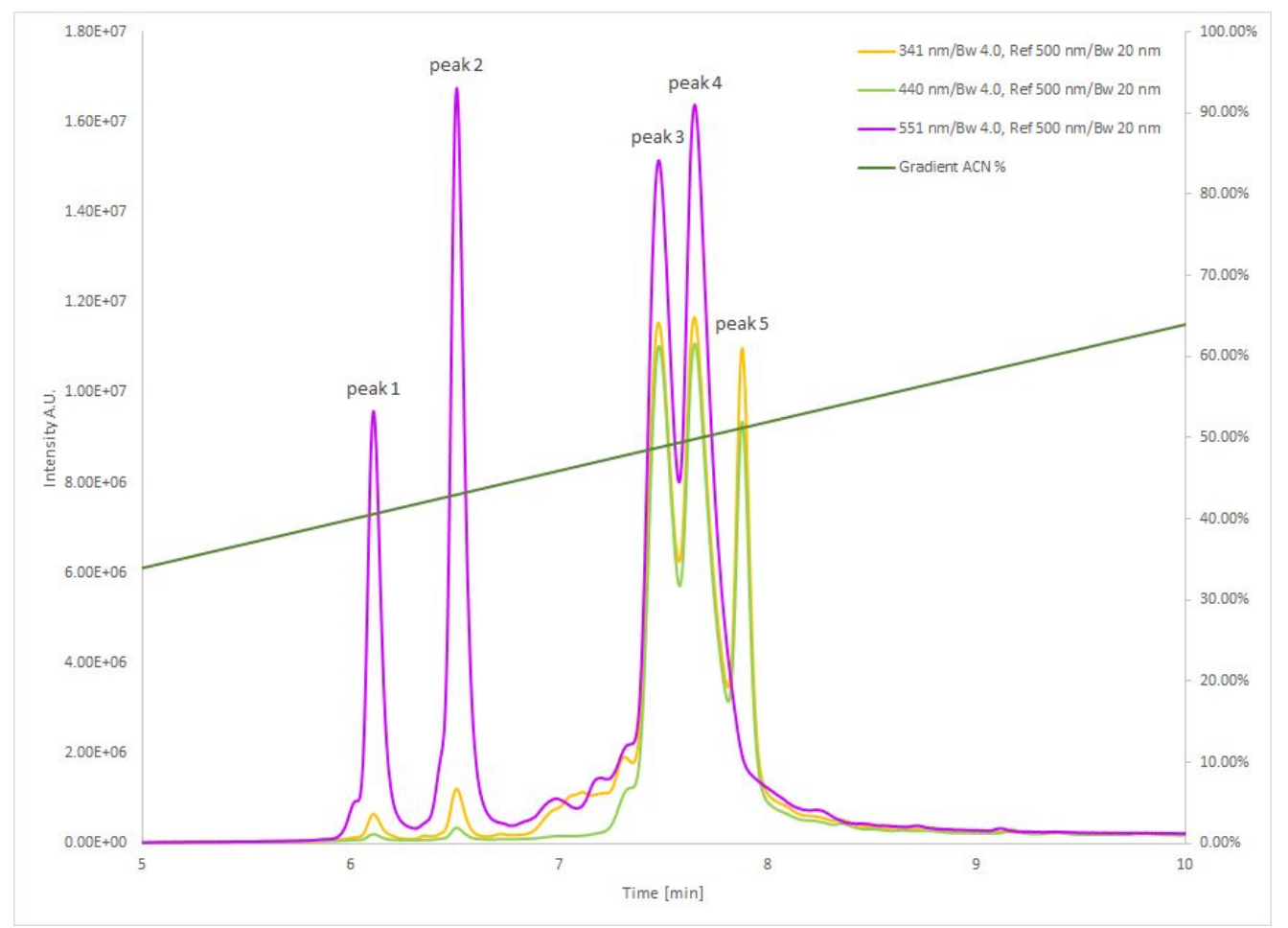

Figure S12. Zoom (5-10 min) of analytical HPLC run of cleaved H-NE. Gradient $10-100 \%$ acetonitrile in water with formic acid $(0.1 \% \mathrm{v} / \mathrm{v})$. The 5(6)-TAMRA isomer accounts for the double peak. Wavelengths for detection $341 \mathrm{~nm}$ (Hoechst), $440 \mathrm{~nm}$ (Coumarin343) and $551 \mathrm{~nm}$ (TAMRA). Peak1 and 2 double peak of acceptor fragment with 5(6)TAMRA absorbance, peak 3 and 4 double peak of intact $H-N E$ and peak 5 single peak of donor fragment with coumarin343 and Hoechst absorbance. 


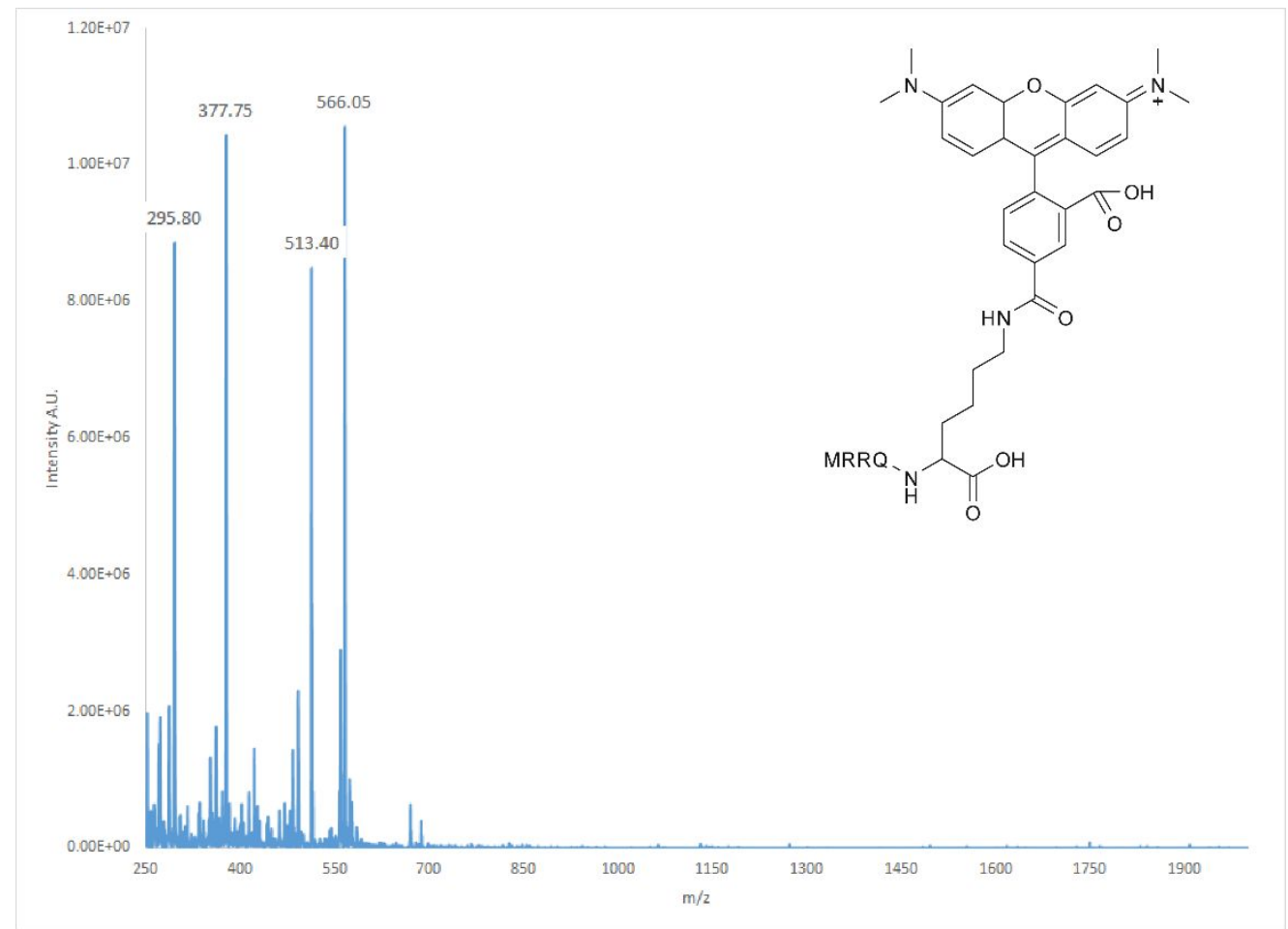

Figure S13. Mass spectrum of peak 1 and 2 (full peak area) of cleaved H-NE HPLC. Calculated mass of acceptor fragment: $[M+1]^{+}=1130.57,[M+2]^{2+}=565.79,[M+3]^{3+}=377.53$, $[M+4]^{4+}=283.40$. 


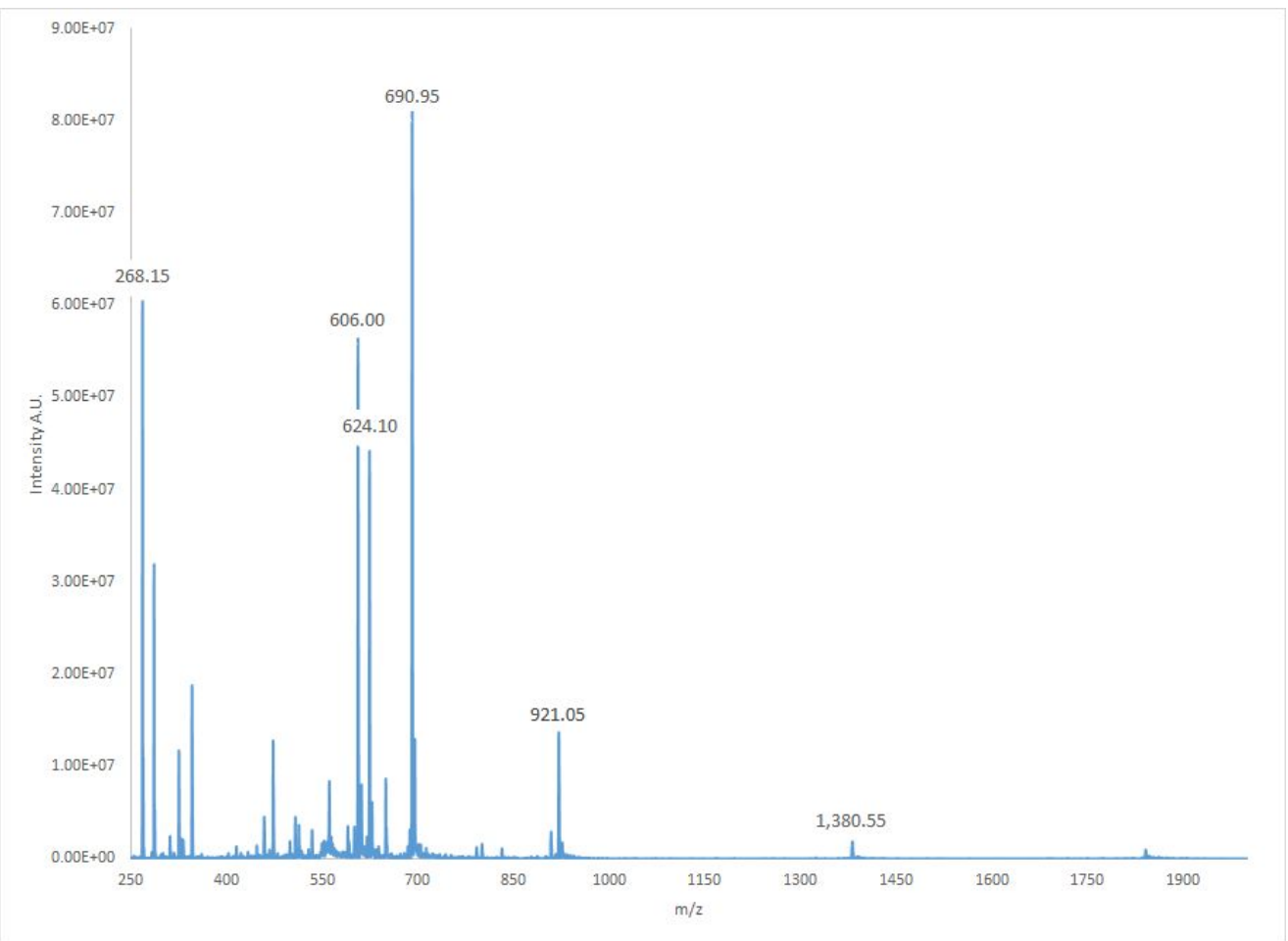

Figure S14. Mass spectrum of peak 3 and 4 (full peak area) of cleaved H-NE HPLC. Calculated mass of $\mathrm{H}-\mathrm{NE}:[\mathrm{M}+1]^{+}=2758.31,[\mathrm{M}+2]^{2+}=1379.66,[\mathrm{M}+3]^{3+}=920.11,[\mathrm{M}+4]^{4+}=$ 690.33, $[M+5]^{5+}=552.47$. 


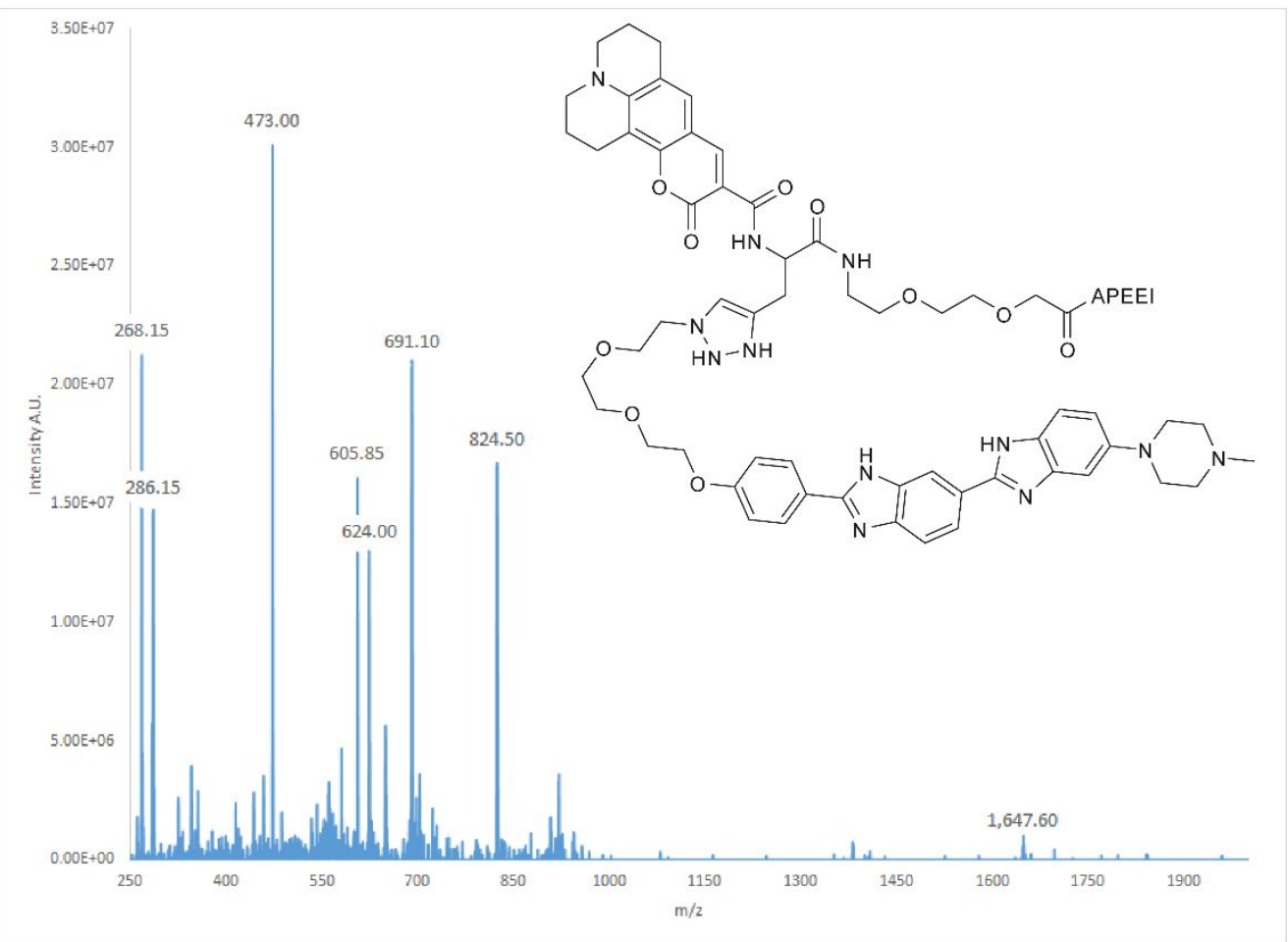

Figure S15. Mass spectrum of peak 5 (full peak area) of cleaved H-NE HPLC.

Calculated mass of donor fragment: $[M+1]^{+}=1646.76,[M+2]^{2+}=823.89,[M+3]^{3+}=549.59$, $[M+4]^{4+}=412.45$. 


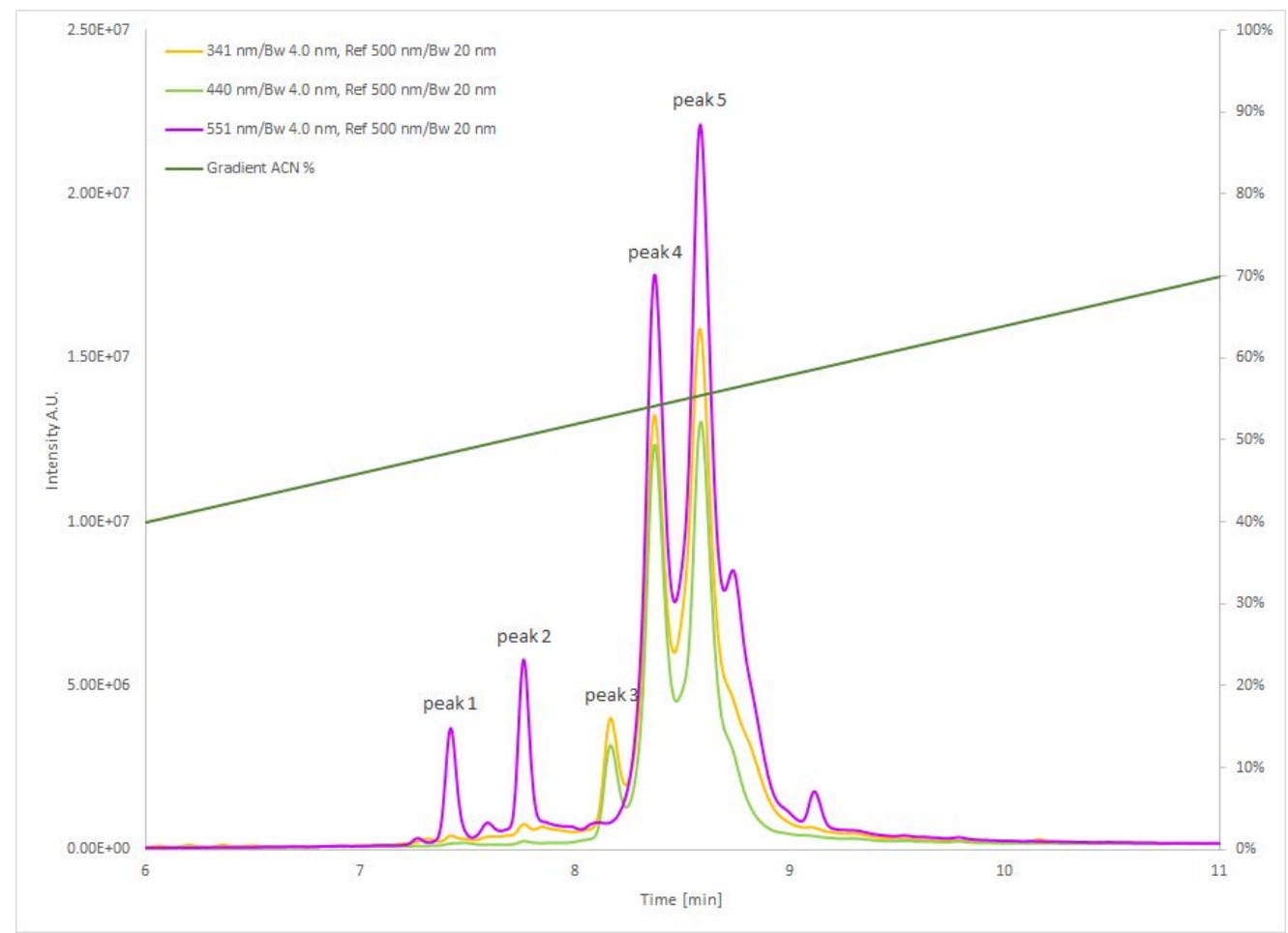

Figure S16. Zoom (6-11 min) of analytical HPLC run of cleaved H-CG. Gradient $10-100 \%$ acetonitrile in water with formic acid $(0.1 \% \mathrm{v} / \mathrm{v})$. The 5(6)-TAMRA isomers account for the double peak. Wavelengths for detection $341 \mathrm{~nm}$ (Hoechst), $440 \mathrm{~nm}$ (Coumarin343) and $551 \mathrm{~nm}$ (TAMRA). Peak1 and 2 double peak of acceptor fragment with TAMRA absorbance, peak 3 single peak of donor fragment with coumarin343 and Hoechst absorbance and peak 4 and 5 double peak of intact $H-C G$. 


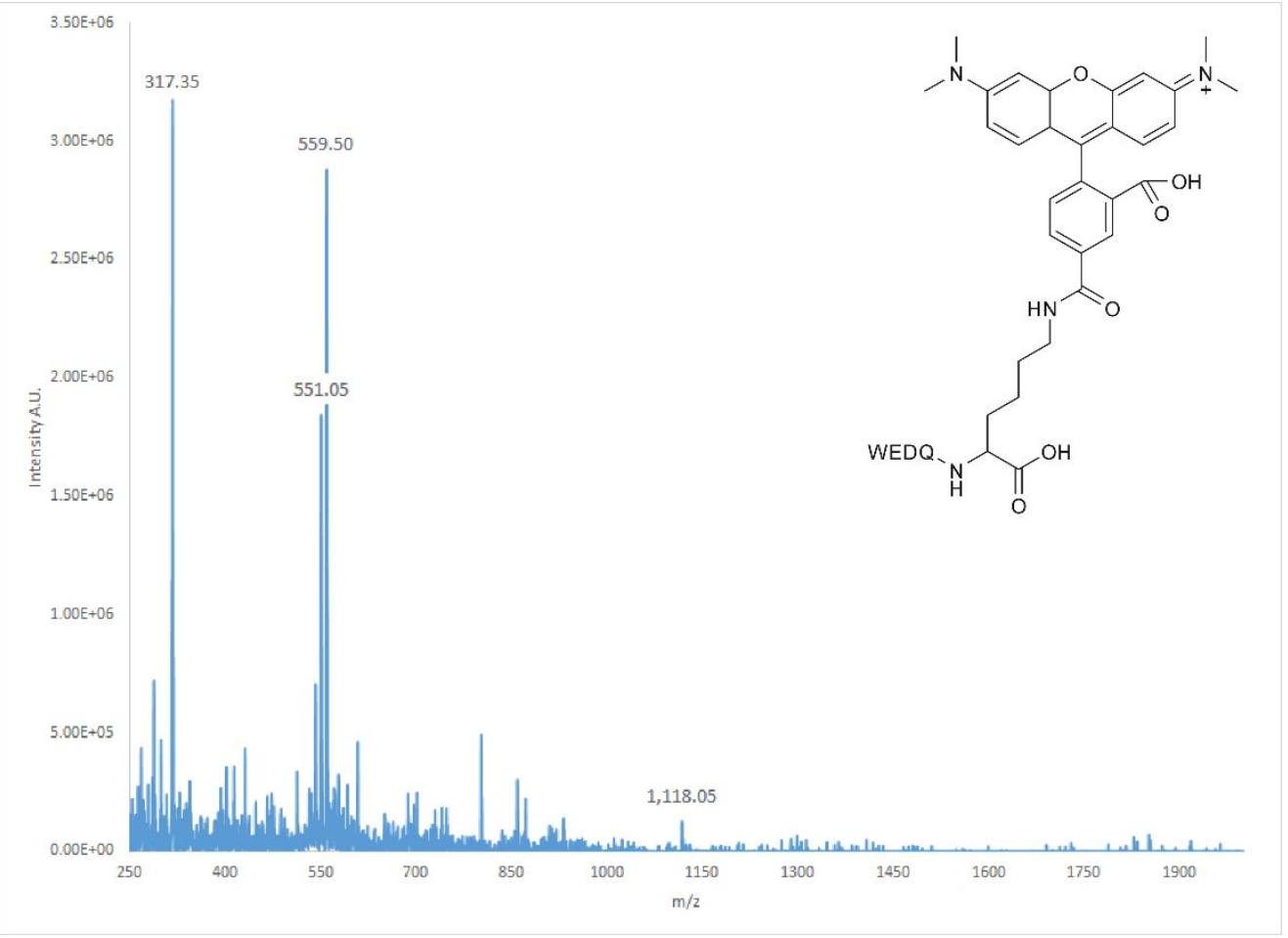

Figure S17. Mass spectrum of peak 1 and 2 (full peak area) of cleaved H-CG HPLC. Calculated mass of acceptor fragment: $[M+1]^{+}=1117.47,[M+2]^{2+}=559.24,[M+3]^{3+}=373.16$, $[M+4]^{4+}=280.12$. 


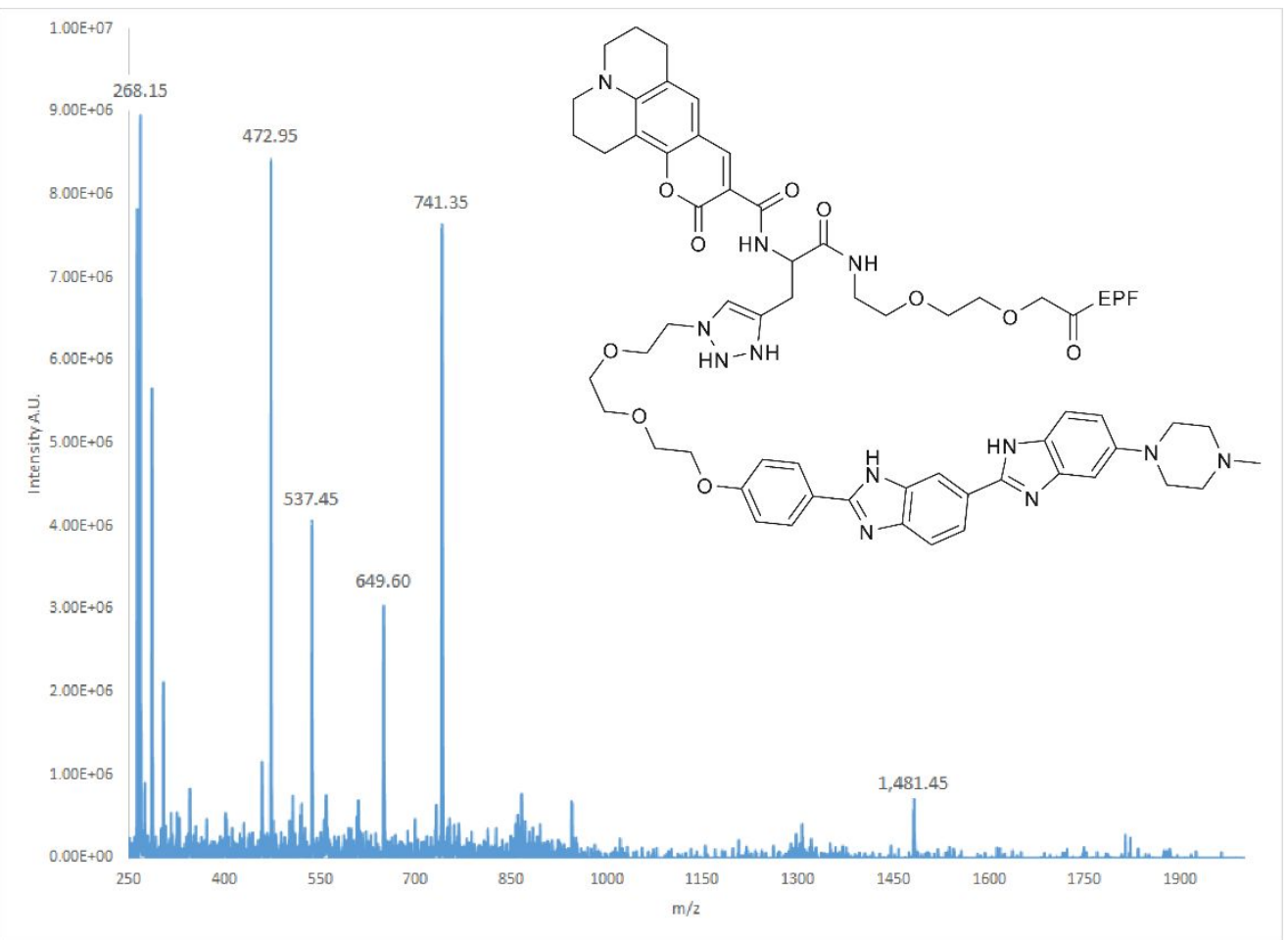

Figure S18. Mass spectrum of peak 3 (full peak area) of cleaved H-CG HPLC.

Calculated mass of donor fragment: $[M+1]^{+}=1480.67,[M+2]^{2+}=740.84,[M+3]^{3+}=494.23$, $[M+4]^{4+}=370.92$. 


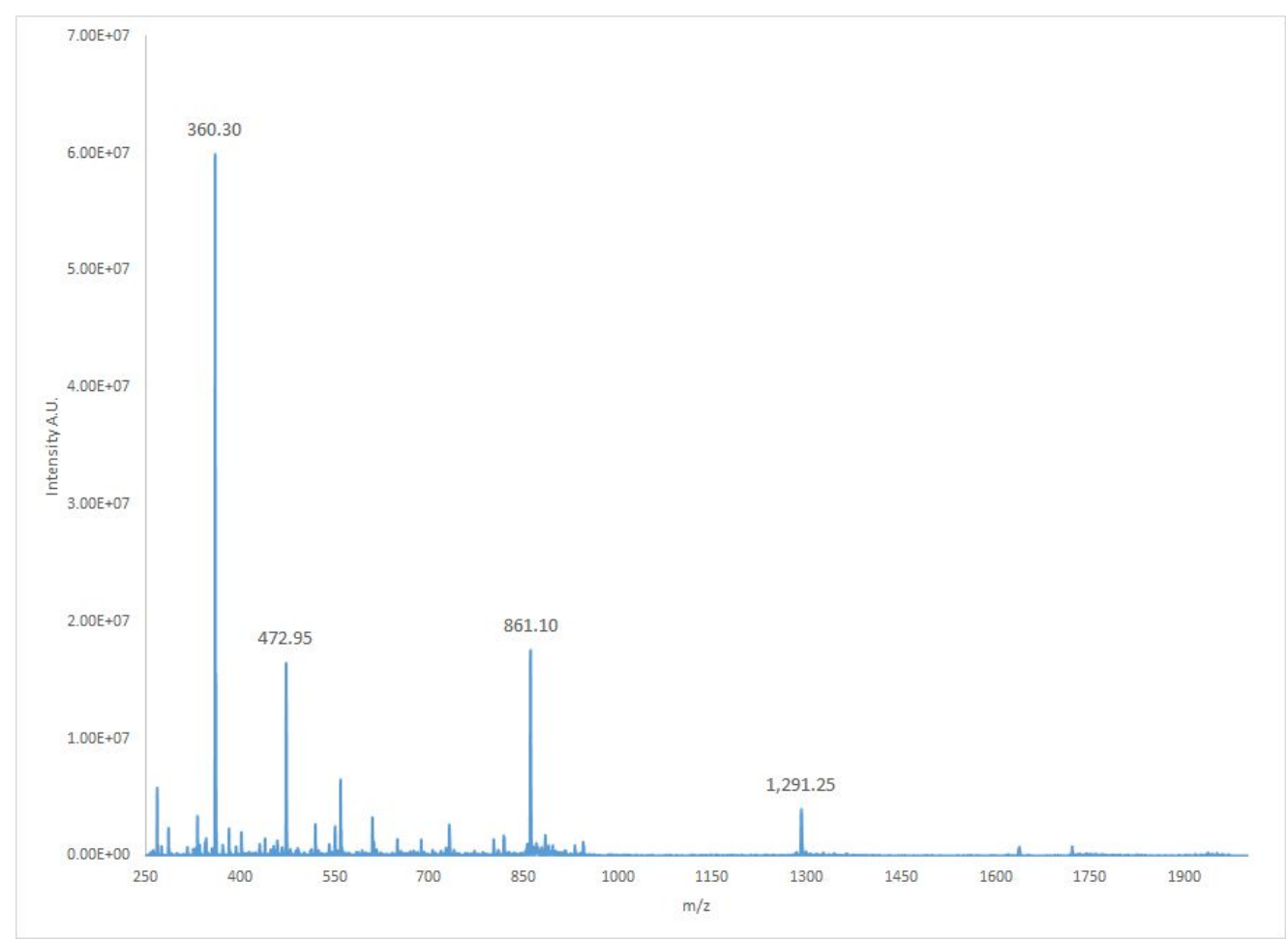

Figure S19. Mass spectrum of peak 4 and 5 (full peak area) of cleaved H-CG HPLC. Calculated mass of $\mathrm{H}-\mathrm{CG}:[M+1]^{+}=2579.12,[M+2]^{2+}=1290.07,[M+3]^{3+}=860.38,[M+4]^{4+}=$ 645.54.

\section{Experimental procedures}

\section{In vitro measurements}

All in vitro experiments were performed with an Enspire 2300 plate reader (PerkinElmer, Waltham, $\mathrm{MA}, \mathrm{USA}$ ) at $25^{\circ} \mathrm{C}$. For absorbance measurements, all probes [100 $\mu \mathrm{M}$ ] were dissolved in TBS and absorbance was recorded between $300 \mathrm{~nm}$ and $700 \mathrm{~nm}$ in white 96-well half area microplate (Greiner, Cat. No.: 675095). Enzymatic assays were carried out in $100 \mathrm{mM}$ TRIS-HCl, $500 \mathrm{mM}$ $\mathrm{NaCl}(\mathrm{pH} 7.5)$ at room temperature (RT) and reporter concentrations were kept at $2 \mu \mathrm{M}$. Measurements were carried out in 96 well half area assay plates (Corning, Cat. No.: 3686) When $\mathrm{H}-\mathrm{NE}$ and $\mathrm{H}-\mathrm{CG}$ were tested, they were incubated with a solution of $50 \mu \mathrm{M}$ of a hairpin forming DNA (5'-CGC GAA TTC GCG TTT TCG CGA ATT CGC G-3', Microsynth), which was previously boiled for $10 \mathrm{~min}$ and cooled down to RT. Then, $10 \mu \mathrm{L}$ of the solution containing $10 \mu \mathrm{M}$ of reporter and $50 \mu \mathrm{M}$ of DNA in TBS was added to wells containing $40 \mu \mathrm{L}$ of enzyme (in $100 \mathrm{mM} \mathrm{TRIS}-\mathrm{HCl}$, $500 \mathrm{mM} \mathrm{NaCl}, \mathrm{pH}$ 7.5) right before fluorescence recording. All enzymes were assayed at a concentration of $100 \mathrm{nM}$, with the exception of mouse enzymes, for which the final concentration was $400 \mathrm{nM}$, and $P$. aeruginosa supernatant, for which a 1:10 dilution in $100 \mathrm{mM}$ TRIS-HCl, 500 $\mathrm{mM} \mathrm{NaCl}$ ( $\mathrm{pH} 7.5$ ) was performed prior to measurement. $P$. aeruginosa (PAO1) was grown in LB medium to $O D=3$ and supernatant was separated via two consecutive centrifugation steps. Representative fluorescence spectra were obtained via reporter excitation at $450 \mathrm{~nm}$ wavelength and signal recording between 470 and $650 \mathrm{~nm}$ ( $3 \mathrm{~nm}$ slit size). For specificity measurements, $\mathrm{H}$ - 
$\mathrm{NE}$ and $\mathrm{H}-\mathrm{CG}$ were excited with $450 \mathrm{~nm}$ wavelength, and donor and acceptor emission were recorded at 500 and $600 \mathrm{~nm}$, respectively. Measurements were carried out for 40 minutes (20 time points, 120 seconds apart). All specificity experiments were repeated at least three times and a minimum of three technical replicates were recorded. To confirm that the employed enzymes were active, NE and PR3 activity was tested with the commercial substrate NMethoxysuccinyl-Ala-Ala-Pro-Val p-nitroanilide (Cat. No.: 454454). CG and CHY activity was confirmed with the N-Succinyl-Ala-Ala-Pro-Phe p-nitroanilide substrate (Cat. No.: S0448). MMP12 activity was confirmed with the Mca-PLGL-Dpa-AR-NH2 fluorogenic substrate (Cat. No.: ES001) and CS activity with the Mca-RPKPVE-Nval-WRK(Dnp)-NH2 fluorogenic substrate (Cat. No.: ES002). The commercial substrates final concentration was selected according to manufacturer's protocol, while enzyme final concentration was $100 \mathrm{nM}$.

The reporter specificity was calculated by comparing the linear regression slope of donor/acceptor increase over time in presence of different enzymes or bacteria supernatants. The slope of a linear regression model indicates the rate of change in $y$ (donor/acceptor ratio) as $x$ (time) changes, therefore we utilize this coefficient as the cleavage rate for a given enzyme.

\section{Blood neutrophils purification and stimulation.}

Human blood was collected from healthy volunteers in $5 \mathrm{~mL}$ S-Monovette ${ }^{\circledR}$ citrate tubes (Sarstedt, Cat. No.: 05.1071). Afterwards, neutrophils were isolated (purity $>95 \%$ ) from $8 \mathrm{~mL}$ of whole blood via the MACSxpress ${ }^{\circledR}$ Whole Blood Neutrophil Isolation Kit (Miltenyi Biotec, Cat. No.: 130-104-434) following manufacturer's protocol. Once purified, neutrophils were resuspended in medium containing RPMI 1640 (Gibco, Karlsruhe, Germany) supplemented with 10\% (v/v) heat inactivated FBS (Gibco, Karlsruhe, Germany) and $0.01 \mathrm{mg} / \mathrm{ml}$ Primocin (Invitrogen, Karlsruhe, Germany). 75000 to 150000 neutrophil were seeded on $12 \mathrm{~mm}$ round glass coverslips laid at the bottom of 24 well plates (Corning ${ }^{\circledR}$ Costar $®$ Multiple Well Plates, Cat. No.: 3526, Cornig Incorporated) for $45 \mathrm{~min}$ to 1 hour at $37^{\circ} \mathrm{C}, 5 \% \mathrm{CO}_{2}$.

\section{H-NE localization in human neutrophils}

After seeding, cells were washed once with PBS, then fixed in $500 \mu \mathrm{L}$ PFA (Carl Roth, Karlsruhe, Germany) ( $4 \%$ in PBS) for 20 min at $37^{\circ} \mathrm{C}$. Fixed cells were washed one time with PBS and permeabilized with $500 \mu \mathrm{L}$ of TritonX-100 (Cat. No.: A4975, PanRean AppliChem) for 15 min at RT. Afterwards, cells were washed twice with PBS and incubated with a solution of PBS containing $2 \mu \mathrm{M}$ of reporter for $30 \mathrm{~min}$ at $37^{\circ} \mathrm{C}$. Finally, cells were washed once with PBS and twice with $\mathrm{ddH}_{2} \mathrm{O}$, dried and mounted with ROTIßHistokit (Carl Roth, Karlsruhe, Germany). The quantification of the reporter signal inside the cell was performed via segmenting manually the cells in the brightfield channel and measuring the fluorescence intensity in the selected area. This experiment was performed twice with blood neutrophils isolated from two blood donors. Technical replicates from each donor were prepared and imaged.

\section{NETs experiments}

To induce NETs, neutrophils were stimulated with phorbol 12-myristate 13-acetate (PMA) (Sigma Aldrich, Cat. No.: P8139) at a concentration of $2 \mu \mathrm{M}$ for 2.5 hours at $37^{\circ} \mathrm{C}, 5 \% \mathrm{CO}_{2}{ }^{1}$.

- To determine the Hoechst-azide compound spillover into the reporters' channel, after seeding, NETs were washed twice with PBS, then incubated with a PBS solution containing either Hoechst-azide or $\mathrm{H}-\mathrm{CG}\left[2 \mu \mathrm{M}\right.$ ] for $30 \mathrm{~min}$ at $37^{\circ} \mathrm{C}$, followed by washing 1 time with PBS, 2 times with $\mathrm{dd}_{2} \mathrm{O}$, drying and mounting with ROTI@Histokit (Carl Roth, 
Karlsruhe, Germany). Line profile analysis was performed with the Plot Profile function in FIJI (V1.51g). Experiments were performed twice and technical duplicates were imaged.

- To prove the colocalization between H-NE and Draq5 (Thermo Fisher, Cat. No.: 62251), cells were washed once with PBS after PMA stimulation. After resuspending in $500 \mu \mathrm{L}$ of PBS, Draq5 was added to a final concentration of $400 \mathrm{nM}$ and either A-NE or H-NE were added to a final concentration of $2 \mu \mathrm{M}$ per well. Dyes were incubated with NETs for 30 min at $37^{\circ} \mathrm{C}, 5 \% \mathrm{CO}_{2}$. Finally, NETs were fixed with PFA (Carl Roth, Karlsruhe, Germany) (4\% in PBS) for 20 min at $37^{\circ} \mathrm{C}$ and washed once with PBS and twice with $\mathrm{ddH}_{2} \mathrm{O}$, dried and mounted with ROTIBHistokit (Carl Roth, Karlsruhe, Germany). Experiments were performed form NETs derived from two donors and technical duplicates were imaged and analyzed. Representative images were acquired via 3D tilescan acquisition. Line profile analysis were performed via Plot Profile function in FIJI (V1.51g) and Pearson correlation coefficient and area overlap were calculated with the FIJI plug-in FluoQ (V3-97)2. For live cells experiments, imaging was started right after PMA [2 $\mu \mathrm{M}]$, Draq5 [400 nM] and H-NE $[2 \mu \mathrm{M}]$ addition.

- To demonstrate that H-NE detects NE activity when bound to DNA, NETs were washed twice with PBS after seeding and stimulation. Afterwards, different concentrations of NE $\left[1,5,10,100\right.$ and $200 \mathrm{nM}$ ] were exogenously added for $30 \mathrm{~min}, 37^{\circ} \mathrm{C}, 5 \% \mathrm{CO}_{2}$. The "0" condition indicates NETs to which no enzyme was added and incubated with Sivelestat (Tocris Bioscience, Cat. No.: 3535) [100 $\mu \mathrm{M}]$ for the duration of the experiment, to keep the donor/acceptor increase at its minimum. NETs were then washed twice with PBS to eliminate excess of enzyme, resuspended in $500 \mu \mathrm{L}$ of PBS and incubated with $2 \mu \mathrm{M}$ of $\mathrm{H}$ $\mathrm{NE}$ for $30 \mathrm{~min}$ at $37^{\circ} \mathrm{C}, 5 \% \mathrm{CO}_{2}$. Eventually, NETs were fixed with PFA (Carl Roth, Karlsruhe, Germany) (4 \% in PBS) for 20 min at $37^{\circ} \mathrm{C}$ and washed once with PBS and twice with $\mathrm{ddH}_{2} \mathrm{O}$, dried and mounted with $\mathrm{ROTI} \otimes H$ Histokit (Carl Roth, Karlsruhe, Germany). Experiments were performed form NETs derived from three human subjects and technical duplicates were imaged and analyzed. Donor/acceptor values were calculated on manually segmented NETs via the FIJI plug-in FluoQ (V3-97)2.

- To measure DNA-bound NE and CG activity on blood neutrophils derived NETs, NETs were washed one time with PBS after seeding and stimulation. Afterwards, NETs were resuspended in $500 \mu \mathrm{L}$ of PBS and either H-NE or H-CG $[2 \mu \mathrm{M}]$ were added for different time windows (5 min to 2 hours). As a negative control, either Sivelestat (Tocris Bioscience, Cat. No.: 3535) or cathepsin G inhibitor I (Merck Millipore, Cat. No.: 219372) [100 $\mu \mathrm{M}]$ were incubated for $20 \mathrm{~min}$ at $37^{\circ} \mathrm{C}$ and $5 \% \mathrm{CO}_{2}$ prior to reporter incubation. Serving as a positive control, human neutrophil elastase (Elastin Product Company, Cat. No.: SE563) or cathepsin G (Elastin Product Company, Cat. No.: SG623) [800 nM] were added right before reporter addition. Eventually, NETs were fixed with PFA (Carl Roth, Karlsruhe, Germany) (4\% in PBS) for 20 min at $37^{\circ} \mathrm{C}$ and washed once with PBS and twice with $\mathrm{ddH}_{2} \mathrm{O}$, dried and mounted with $\mathrm{ROTI} \otimes H$ istokit (Carl Roth, Karlsruhe, Germany). Experiments were performed form NETs derived from four (H-NE experiments) and three (H-CG experiments) human donors and technical duplicates were imaged and analyzed. Donor/acceptor values were calculated on manually segmented NETs via the FIJI plug-in FluoQ (V3-97)². 
- To demonstrate NE and CG presence on NETs via immunocytochemistry, after seeding and PMA (Sigma Aldrich, Cat. No.: P8139) stimulation, NETs were fixed with a solution of 4\% PFA (Carl Roth, Karlsruhe, Germany) in PBS for 30 min at RT. NETs were then washed 1x with PBS and permeabilized with Triton X-100 0.1\% (Cat. No.: A4975, PanRean AppliChem) for $10 \mathrm{~min}$ at RT, then washed 2x PBS. After, blocking solution (Block Aid $^{\mathrm{TM}}$, Thermo fisher, Cat. No.: B10710) was added to each well at RT for $30 \mathrm{~min}$. Then the mouse primary antibody anti human ELA2 (R\&D, Cat. No.: MAB91671, clone number: $950317,10 \mu \mathrm{g} / \mathrm{mL}$ final concentration, diluted in blocking solution) or the rabbit primary antibody anti human cathepsin G (Invitrogen, Cat. No.: 703590, clone number: 12H15L69, $10 \mu \mathrm{g} / \mathrm{mL}$ final concentration, diluted in blocking solution) were added and incubated for 1 hour at RT. NETs were then washed 3 times with PBS and the anti-mouse secondary antibody (anti-mouse lg1, Invitrogen, Cat. No.: A21240, conjugated with Alexa Fluor 647, diluted 1:150 in blocking solution) or the anti-rabbit secondary antibody (antirabbit IgG, Thermo Fisher, Cat. No.: A21245, conjugated with Alexa Fluor 647, diluted 1:150 in blocking solution) were added for 1 hour in dark at RT. Finally, cells were washed 3x with PBS, Hoechst 33342 (1:12500 dilution) (Cat. No.: 62249, Thermo Fisher) was incubated for 5 min and wells washed one last time $2 x$ with PBS and $2 x$ with distilled water before drying, and mounting. Immunofluorescence negative controls were performed by incubating NETs with secondary antibodies only, and no fluorescence signal was detected on NETs when such control was performed.

\section{Cystic fibrosis sputum processing and experiments}

All human samples were collected upon signed written informed consent, which was approved by the Ethics Committee of the University Hospital Heidelberg. The induced sputum was collected in a petri dish. Four volumes of $10 \%$ Sputolysin solution (Calbiochem, Darmstadt, Germany) were added to sputum to dissolve mucins and the mixture was mildly shaken for 15 min at room temperature. The mixture was then diluted by adding the same volume of cold PBS and filtered twice through 100 and $40 \mu \mathrm{m}$ cell strainers. Finally, the solution was centrifuged at $300 \mathrm{~g}$ and $4^{\circ} \mathrm{C}$ for $10 \mathrm{~min}$. Cell pellets were resuspended in PBS and counted. 30000 cells were resuspended in $50 \mu \mathrm{L}$ of PBS in $1.5 \mathrm{~mL}$ Eppendorf tubes and incubated with a solution of $50 \mu \mathrm{L}$ of PBS containing $2 \mu \mathrm{M}$ of $\mathrm{H}-\mathrm{NE}$. As negative control, prior to reporter addition, sputum cells were incubated for 30 min at RT with $100 \mu \mathrm{M}$ of Sivelestat (Tocris Bioscience, Cat. No.: 3535). H-NE was incubated for $30 \mathrm{~min}$ and then the reaction was rapidly quenched via addition of $100 \mu \mathrm{L}$ of ice cold PBS containing cOmplete ${ }^{\mathrm{TM}}$ Protease Inhibitor Cocktail (Sigma Aldrich, Cat. No.: 11697498001). Cells were then cytospun on microscopy slides, fixed for 10 min in ice cold methanol ( $\geq 99 \%$, Carl Roth, Karlsruhe, Germany), dried and mounted with ROTI®Histokit (Carl Roth, Karlsruhe, Germany). Histology preparation was carried out via standard H\&E staining.

\section{Confocal microscopy and image analysis}

All microscopy images were acquired using a confocal Leica SP8 microscope (Leica Microsystems, Wetzlar, Germany) equipped with either a PL APO 40x or 63X oil objective. Hoechst 33342 (Cat. No.: 62249, Thermo Fisher) and Hoechst-azide were excited with the 405 UV laser line. Coumarin 343 was excited with the $458 \mathrm{~nm}$ Argon laser and its emission sampled between 470 and $500 \mathrm{~nm}$. 5(6)-TAMRA direct excitation was carried out with the $561 \mathrm{~nm}$ diode pumped solid state (DPSS) laser and sampled between 590 and $630 \mathrm{~nm}$. Sensitized acceptor emission was recorded, upon coumarin 343 excitation, between 590 and $630 \mathrm{~nm}$. The nuclear stain Draq5 and the secondary antibodies employed for immunofluorescence experiments which were coupled with the Alexa Fluor 647 dye were excited with a helium-neon-laser line at $633 \mathrm{~nm}$. 
The pinhole was set at the beginning of image acquisition and kept constant throughout the same experiments. For live cell imaging, an environment chamber (EMBL, Heidelberg) set at $37^{\circ} \mathrm{C}$ and $5 \%$ CO2 was used. To image mouse lung slices, 3D tilescans with an oil immersion 40X objective covering the entire surface of the airway were acquired. Images and movies were analyzed by Imaris (V9.5.1) or FIJI (V1.51g) and the ImageJ macro "FluoQ" (version 3-97)2. The macro operates a background subtraction by ImageJ's built-in rolling ball function, then, it allows the operator to set manually a channel threshold. Finally, images are smoothed via a median filter. Region of interest (ROIs) are selected manually by drawing their border with the pencil tool. When $z$ stacks were processed, the Z-projection of the average intensity of the donor channel was used as channel for cell segmentation. The change in donor/acceptor ratio was then calculated as mean pixel intensity of each ROI (over time in case of time series) from both channels and the ratio between the donor and acceptor was obtained. The same macro was used for colocalization analysis and Pearson correlation coefficient and Area overlap calculation.

\section{Mouse lung slices preparation}

Six weeks old Scnn1b-Tg mice were euthanized via exsanguination after deep anesthesia through intraperitoneal injection of $120 \mathrm{mg} / \mathrm{kg}$ ketamine and $16 \mathrm{mg} / \mathrm{kg}$ xylazine (Sigma-Aldrich) Lungs were removed through a median sternotomy, fixed in $4 \%$ buffered formalin overnight at $4^{\circ} \mathrm{C}$. Fixed lungs were washed in PBS and either stored in $70 \%$ ethanol (Carl Roth, Karlsruhe, Germany) at $4^{\circ} \mathrm{C}$ or directly processed for paraffin embedding. In brief, the apical part of the fixed lungs was cropped transversally and lungs were placed in embedding cassettes (Steinbrenner Laborsysteme, Wiesenbach, Germany) and dehydrated 2 × 30 minutes in $96 \%$ ethanol (Carl Roth, Karlsruhe, Germany), 2 x 45 minutes in $100 \%$ ethanol (Carl Roth, Karlsruhe, Germany), and 12 to 16 hours in xylene (Carl Roth, Karlsruhe, Germany). Samples were then submerged in paraffin (Carl Roth, Karlsruhe, Germany) and vacuum was applied for 2 hours followed by 1 hour at atmospheric pressure. Afterwards, lungs were placed in embedding molds with the cropped surface facing downwards to ensure correct orientation of embedded tissue. Paraffin blocks were kept for 12 to 16 hours at $4^{\circ} \mathrm{C}$ to harden completely. Left lungs were cut with a microtome (Leica Microsystems, Nussloch, Germany) to $5 \mu \mathrm{m}$ and were sectioned transversally at the level of the proximal intra-pulmonary main axial airway near the hilus. Lung sections were deparaffinized in xylene (Carl Roth, Karlsruhe, Germany) and rehydrated 2 x 10 minutes in $100 \%$ ethanol (Carl Roth, Karlsruhe, Germany), 2 x 2 minutes in $96 \%$ ethanol (Carl Roth, Karlsruhe, Germany), 2 minutes in $70 \%$ ethanol (Carl Roth, Karlsruhe, Germany), and finally rinsed in $\mathrm{dd}_{2} \mathrm{O}$. For DNA staining and NE activity quantification serial lung sections of the same mouse were treated with inhibitor, buffer or enzyme to ensure reproducibility and, for direct comparison and minimization of preparation artifacts, the donor/acceptor measured in each slice was normalized to the corresponding inhibitor treated control lung slice. Briefly, deparaffinized and rehydrated lung sections were treated with a PBS solution $(100 \mu \mathrm{L})$ containing either Sivelestat (Tocris Bioscience, Cat. No.: 3535) [1 mM] or human neutrophil elastase (Elastin Product Company, Cat. No.: SE563) [800 nM] or left in $\mathrm{dd}_{2} \mathrm{O}$ for 30 min. After washing with $\mathrm{dd}_{2} \mathrm{O}$, all slides were treated with 100 $\mu \mathrm{L}$ of a PBS solution containing $2 \mu \mathrm{M}$ of $\mathrm{H}-\mathrm{NE}$ for 3 hours. Finally, slices were washed with $\mathrm{dd}_{2} \mathrm{O}$ and mounted with RotiHisto Kit (Carl Roth, Karlsruhe, Germany).

\section{Statistics}

All statistical tests, analysis and plots were performed using $R$ software (R version 3.6.1). 


\section{Supplementary experimental figures}

Figure S20

a<smiles>CN1CCN(c2ccc3[nH]c(-c4ccc5nc(-c6ccc(O)cc6)[nH]c5c4)nc3c2)CC1</smiles><smiles>CN1CCN(c2ccc3[nH]c(-c4ccc5nc(-c6ccc(O)cc6)[nH]c5c4)nc3c2)CC1</smiles>

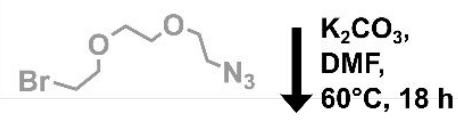<smiles>CN1CCN(c2ccc3[nH]c(-c4ccc5nc(-c6ccc(OCCOCCOCCN)cc6)[nH]c5c4)nc3c2)CC1</smiles>

b<smiles>[R]P=[R]O[Na]</smiles>
$3 \mid \begin{aligned} & \mathrm{Cu}(\mathrm{l}), \\ & \text { TBTA, } \\ & \text { DMSO, } \\ & \mathbf{1 5} \mathrm{h}, \mathrm{RT}\end{aligned}$

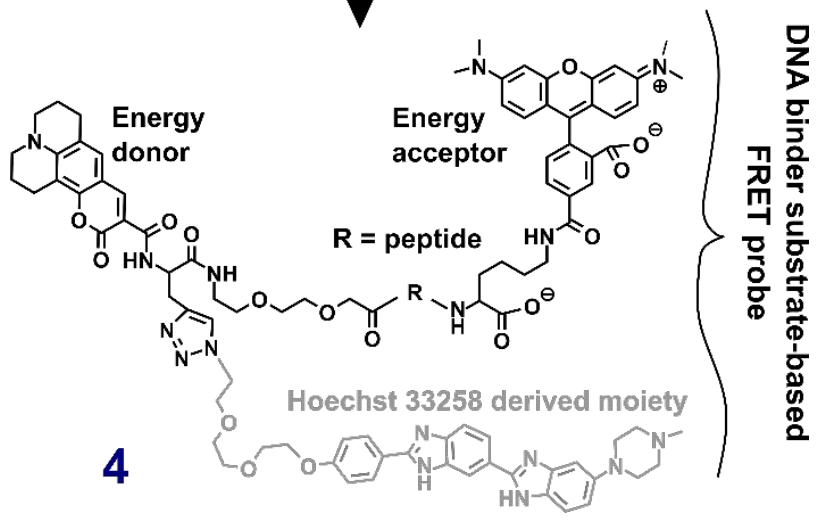

Figure S20. Synthetic route for Hoechst containing-FRET reporters. a) Synthesis of Hoechst-azide (compound 3). b) General synthetic strategy to produce Hoechst-containing reporters from alkyne-containing FRET probe and the Hoechst-azide moiety. 
Figure S21
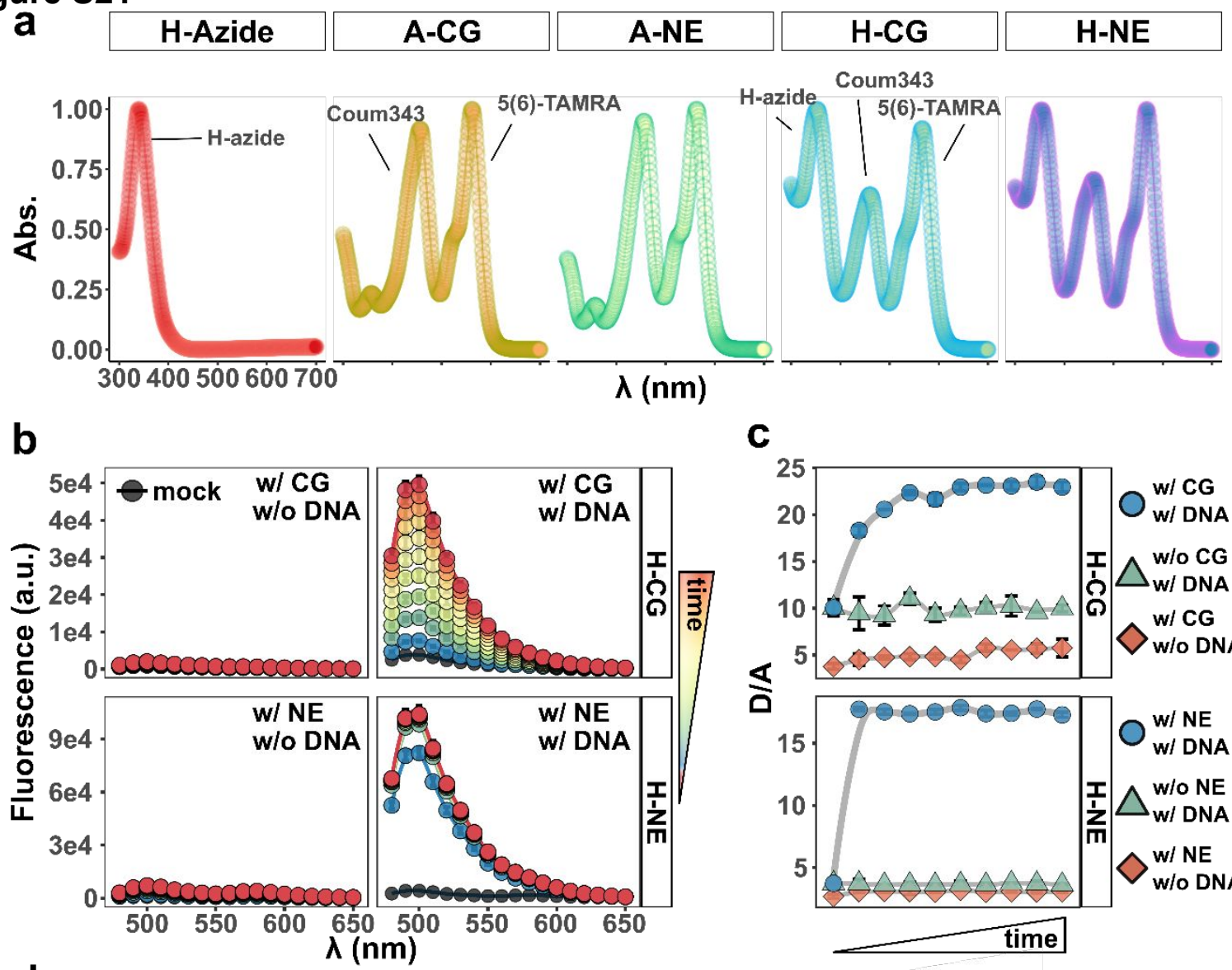

b

C

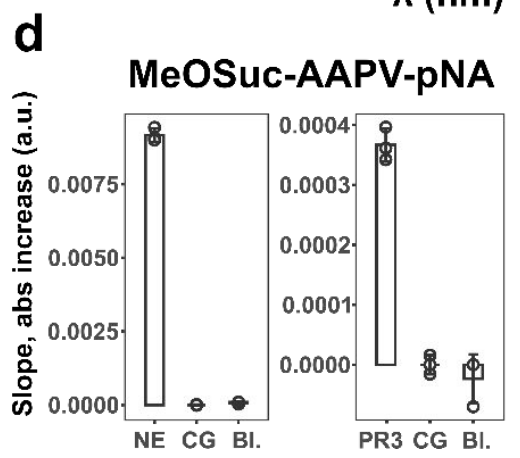

MeOSuc-AAPV-pNA N-Succ-AAPF-pNA
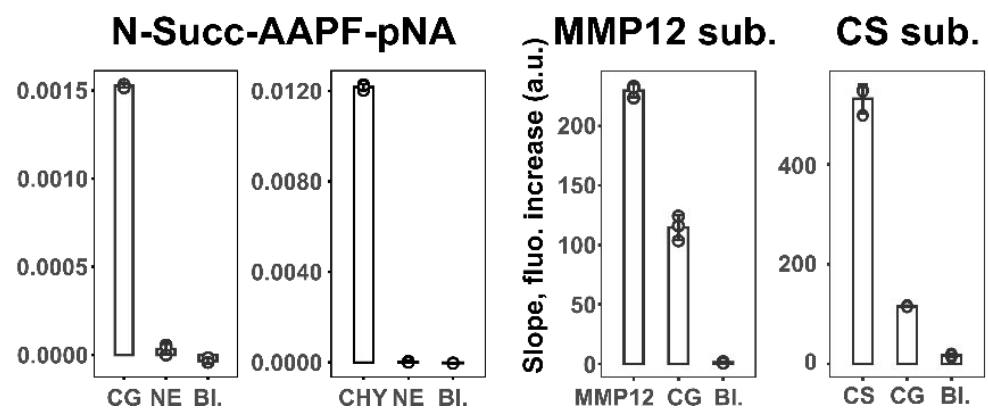

Figure S21. Spectra and cleavage of FRET probes. a) Normalized absorbance measured for Hoechst-azide, A-CG, A-NE, H-CG and H-NE compounds. b) Fluorescence spectra of H-CG and $H$-NE probes incubated with [20 nM] of CG and NE, respectively. On the right side, the probes were added to a solution containing $50 \mu \mathrm{M}$ of a hairpin-forming DNA strand. Fluorescence signal was recorded over $30 \mathrm{~min}$. Data are shown as mean of technical triplicates $\pm S D$. Black points indicate reporters incubated with no enzyme. c) Donor/acceptor increase recorded over $30 \mathrm{~min}$ for H-CG and H-NE incubated with or without CG or NE, respectively, and in presence or absence of exogenous hairpin DNA [50 $\mu \mathrm{M}]$. d) Positive control for enzyme activity. Plots show slopes calculated from absorbance or fluorescence linear increase measured after incubation of commercial substrates with different enzymes. For each plot, the target enzyme, a negative control and buffer only (BI.) were incubated with the commercial peptidic substrate. MMP12 substrate: Mca-PLGL-Dpa-AR-NH ${ }_{2}$, cathepsin S substrate: Mca-RPKPVE-Nval-WRK(Dnp)-NH ${ }_{2}$. Bl.: blank. Data are shown as mean of technical triplicates $\pm S D$. 


\section{Figure S22}

a

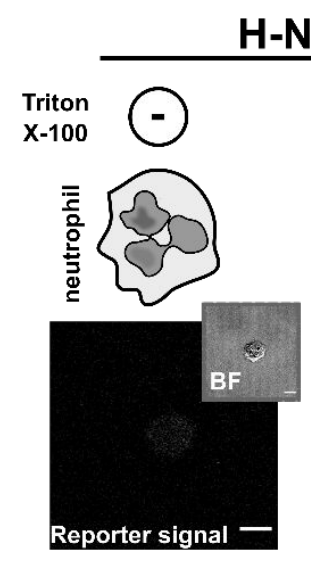

H-NE

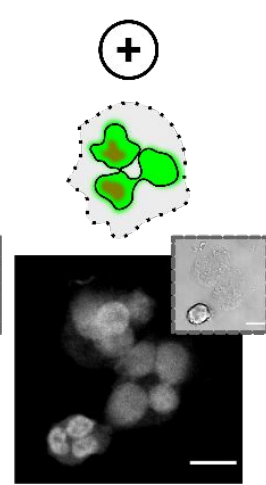

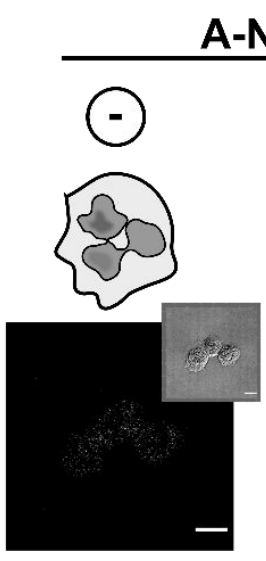

A-NE

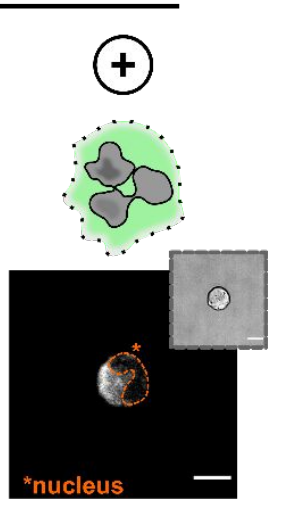

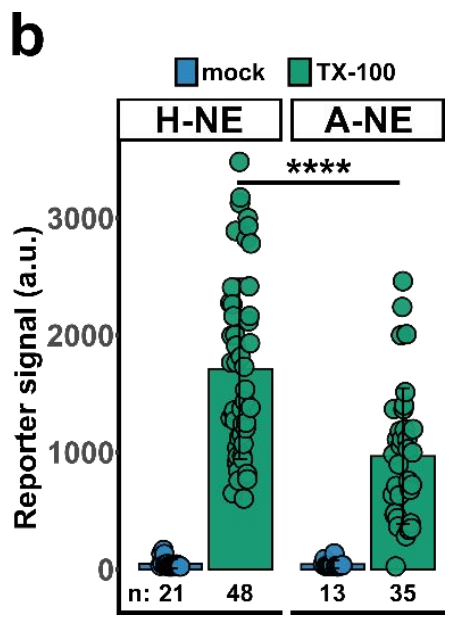

Figure S22. H-NE localization in human neutrophils. a) Confocal microscopy images of blood neutrophils, buffer or Triton X-100 (0.1\% for $10 \mathrm{~min})$ treated and incubated with either $H-N E$ or the control reporter lacking the Hoechst part A-NE. Images show fluorescence recorded in the probes' donor channel. Scale bars: $5 \mu \mathrm{m}$. b) Quantification of intracellular HNE and A-NE signal (donor channel) of mock and Triton X-100 (0.1\% for $10 \mathrm{~min})$ treated blood neutrophils. Experiments were performed on blood neutrophils isolated from two healthy blood donors. Statistics were calculated via Wilcoxon rank sum test. 


\section{Figure S23}

a

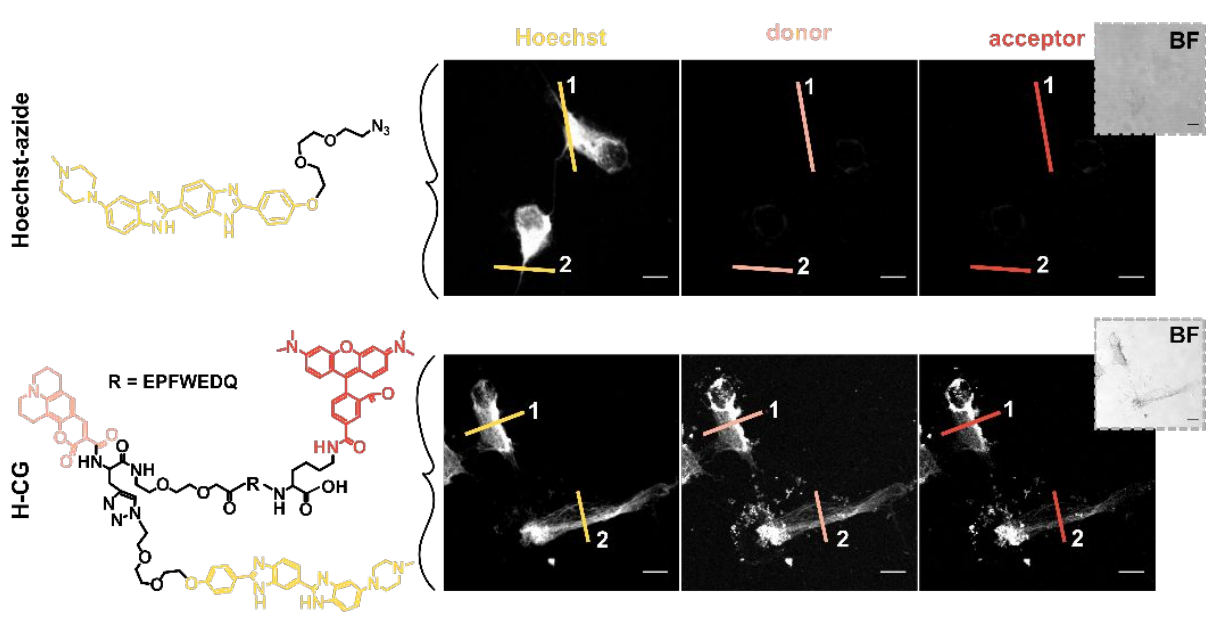

b $\begin{aligned} & \text { Hoechst } \\ & \text { donor }\end{aligned}$

donor

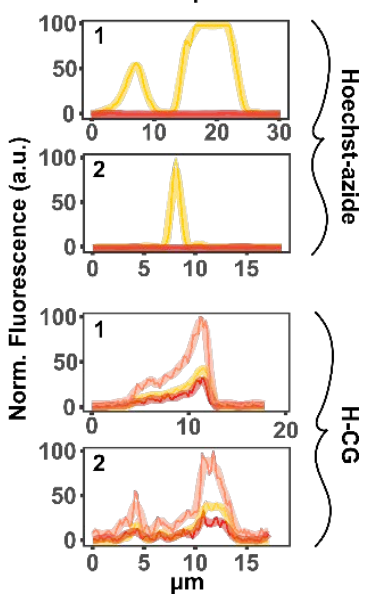

Figure S23. Hoechst azide spillover. a) Confocal microscopy images of PMA (2 $\mu$ M for 2.5 hours) induced NETs derived from blood neutrophils and incubated with either Hoechst azide [2 $\mu \mathrm{M}$ ] or H-CG [2 $\mu \mathrm{M}$ ]. Hoechst, donor (coumarin343) and acceptor (5(6)-TAMRA) channels are shown. Scale bars: $10 \mu \mathrm{m}$. Experiments were performed twice on blood neutrophils derived-NETs obtained from two healthy donors. b) Line plot fluorescence profile of lines shown in (a). Hoechst, coumarin 343 and 5(6)-TAMRA fluorescence signals are plotted. Data are normalized within each plot to the highest fluorescence value measured. 


\section{Figure S24}

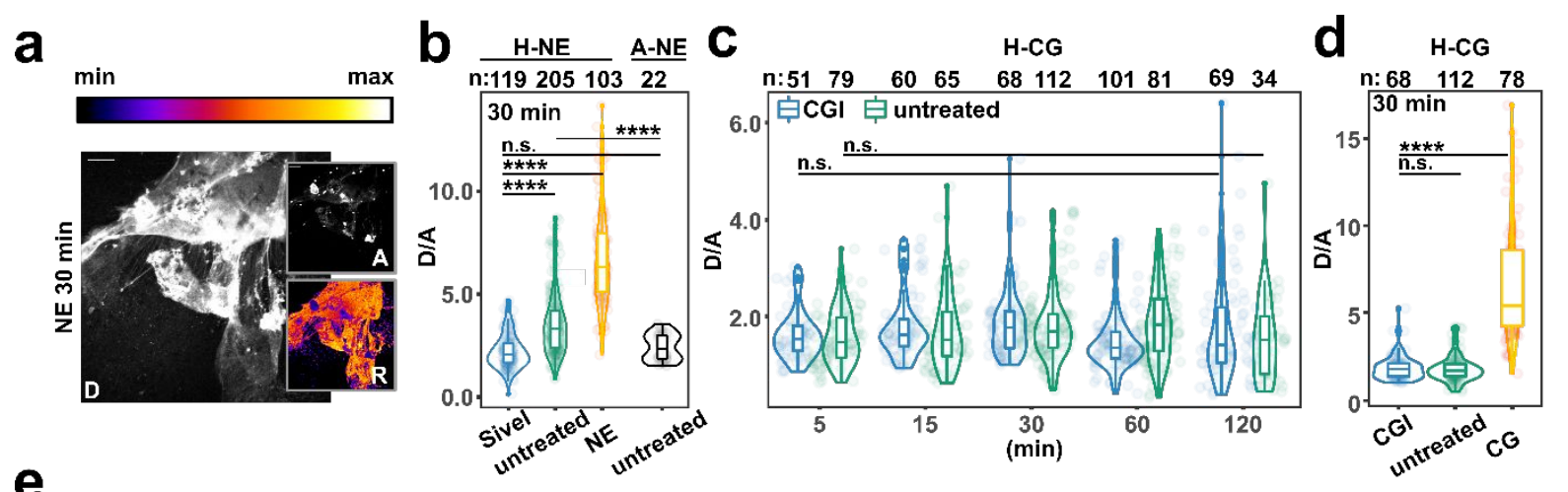

e
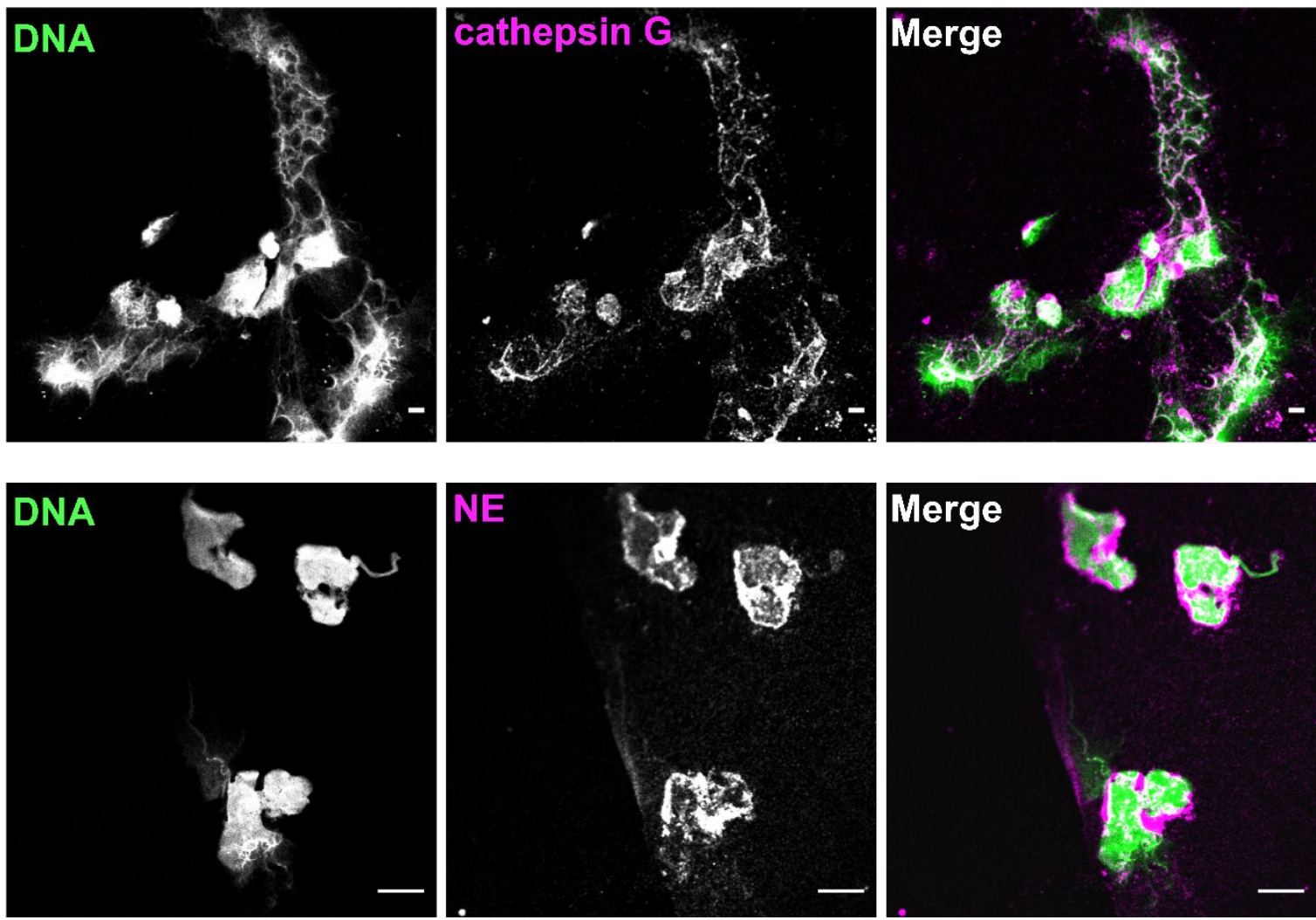

Figure S24. Protease activity on blood NETs. a) Confocal microscopy image showing the donor $(D)$, the acceptor $(A)$ and the donor/acceptor ratio $(R)$ of blood NETs incubated with $H$ NE after NE [800 nM] addition as positive control. Scale bars: $10 \mu \mathrm{m}$. b) Donor/acceptor ratio quantification of blood NETs incubated with H-NE for 30 min after 10 min incubation with Sivelestat [100 $\mu \mathrm{M}]$, left untreated, or incubated for 10 min with NE [800 nM]. The right part of the plot (black data) shows donor/acceptor ratio quantification of blood NETs incubated with A-NE [2 $\mu \mathrm{M}$ ] for 30 min. c) Quantification of CG activity on NETs derived from blood neutrophils isolated from three healthy donors. CGl: cells were preincubated with cathepsin $G$ inhibitor I [100 $\mu \mathrm{M}$ ] for 10 min prior of H-CG addition. d) Donor/acceptor ratio quantification of blood NETs incubated with $\mathrm{H}-\mathrm{CG}[2 \mu \mathrm{M}]$ for 30 min after 10 min incubation with cathepsin $\mathrm{G}$ inhibitor I [100 $\mu \mathrm{M}$ ], left untreated, or incubated for 10 min with CG [800 nM]. Statistics were calculated via Wilcoxon rank sum test. e) Confocal immunofluorescence images of blood NETs stained with Hoechst-33342 (DNA panel) and either cathepsin G or neutrophil elastase antibody. Scale bars: $10 \mu \mathrm{m}$. 


\section{Figure S25}
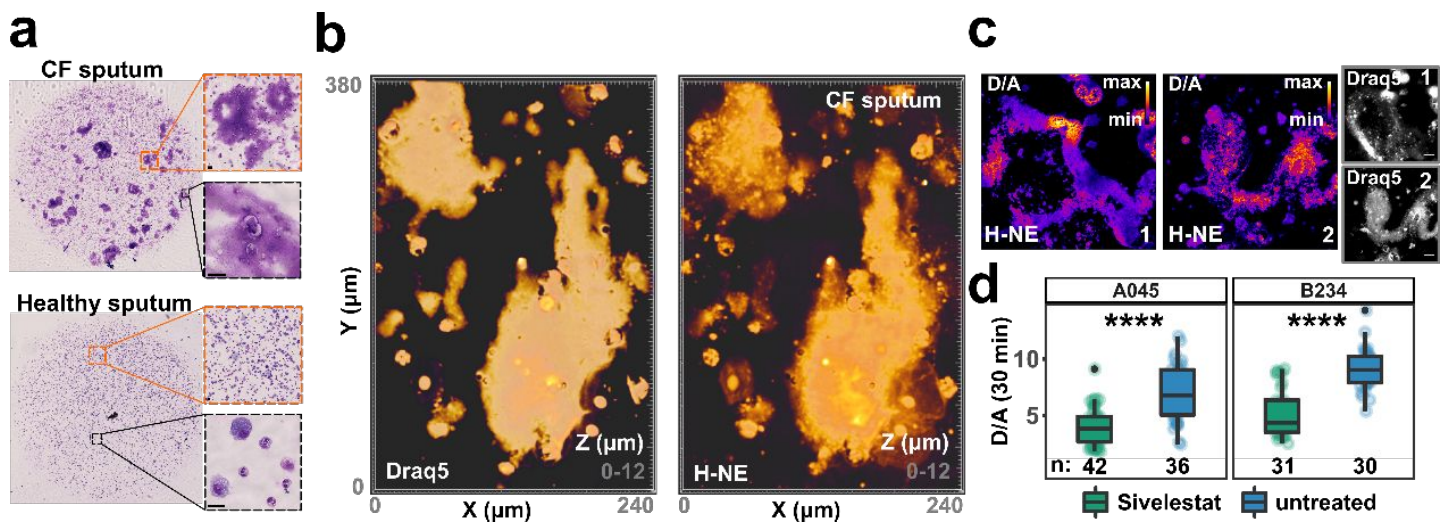

Figure S25. H-NE stains large DNA condensates and reveals high NE activity on CF derived extracellular DNA. a) H\&E stained cytospins of CF (top) and healthy (lower) sputum depicting different amount of extracellular DNA content. Magnified area: segmented nuclei of healthy neutrophils (lower panels) and decondensed and potentially NETing nuclei of CF neutrophils (top panels). b) Confocal 3D image of CF sputum stained with Draq5 (left) and $\mathrm{H}$ NE (donor channel) (right). c) Representative confocal images showing measured NE activity on extracellular DNA present in CF sputum. Scale bars: $10 \mu \mathrm{m}$. d) Boxplots showing quantification of NE activity on CF sputum extracellular DNA, 30 min after addition of H-NE, incubated with or without Sivelestat [100 $\mu \mathrm{M}]$ prior to H-NE addition for 10 min. Patient A045: Sivelestat treated $(D / A=4.00 \pm 1.61)$, untreated $(6.87 \pm 2.46)$. Patient B234: Sivelestat treated $(D / A=5.16 \pm 2.08)$, untreated (9.09 \pm 1.85$)$. Data are derived from 2 CF patients, technical duplicates of each patient and condition were imaged. Statistics were calculated via Wilcoxon rank sum test. 


\section{Figure S26}
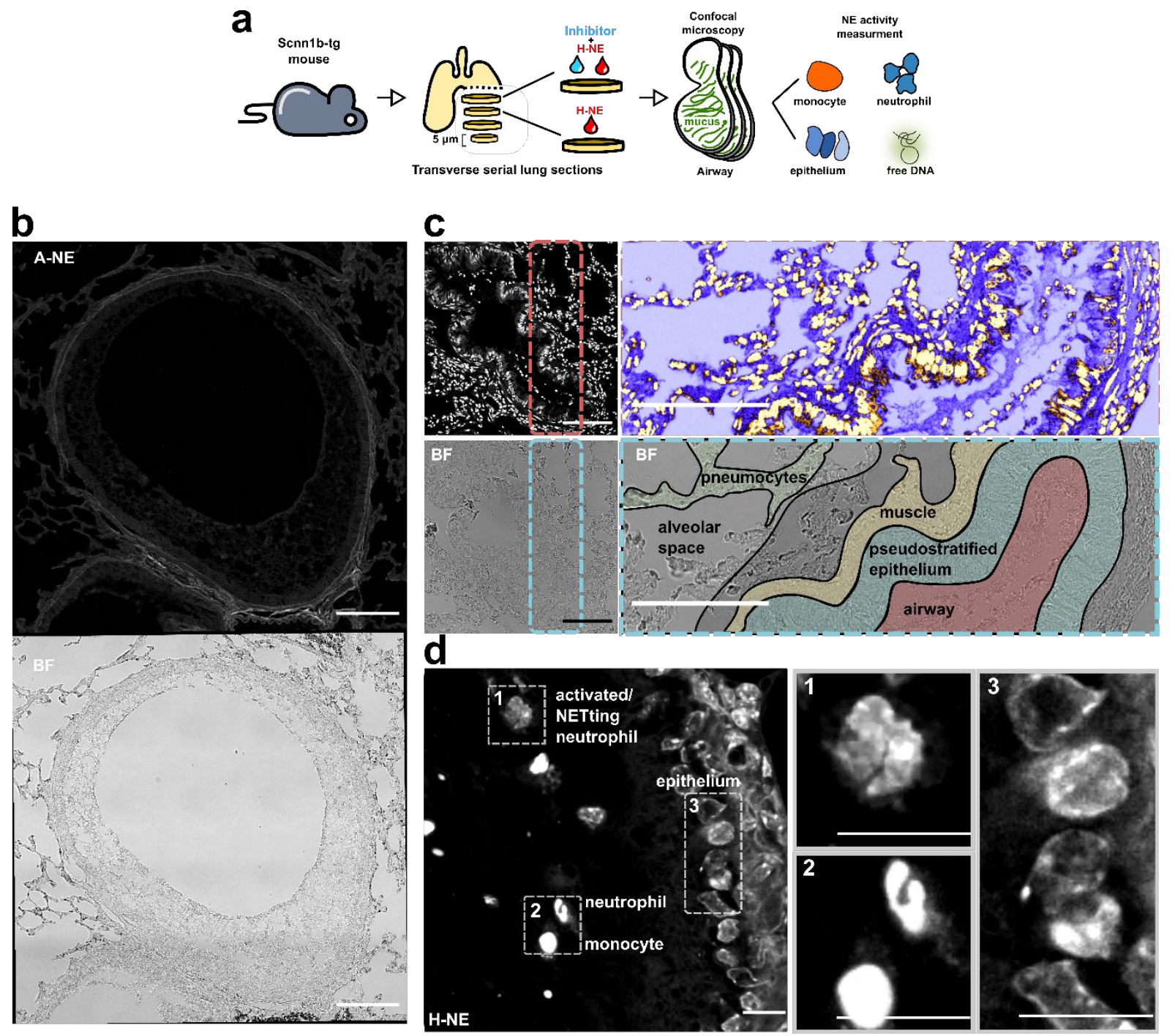

Figure S26. Preparation of CF-like mouse lung slices. a) Schematic representation of the protocol employed to cut, stain and image transverse serial lung slices ( $5 \mu \mathrm{m}$ thick) used to quantify DNA-bound NE activity in tissue. b) Confocal tilescan maximal projection (top) and brightfield (bottom) images of $5 \mu \mathrm{m}$ mouse lung slices stained with $2 \mu \mathrm{M}$ of the control probe ANE (donor channel) for 3 hours. Scale bars: $100 \mu \mathrm{m}$. c) Maximal projection of confocal microscopy tilescan images of lung slices stained with Draq5. On the right side, the major anatomical structures of mouse airways are magnified and highlighted in different colors. Scale bars: $100 \mu \mathrm{m}$. d) Confocal microscopy images of $5 \mu \mathrm{m}$ mouse lung slices stained with H-NE [2 $\mu \mathrm{M}]$ (donor channel) for 3 hours. The major cellular population are magnified and highlighted on the right. Scale bars: $10 \mu \mathrm{m}$. 


\section{Ethical statement and probes availability}

Informed written consent was obtained from all subjects prior to sample collection. Human as well as animal studies were approved by the appropriate ethical committee of Heidelberg University.

The authors are happy to share their new probes upon request. Please contact Matteo Guerra (matteo.guerra@embl.de or 892teo@gmail.com), Victoria S. Halls (hallsv@ohsu.edu) or Carsten Schultz (schulcar@ohsu.edu).

\section{Significant Hazards or Risks Statement}

No unexpected or unusually high safety hazards were encountered.

\section{References}

(1) Brinkmann, V.; Laube, B.; Abu Abed, U.; Goosmann, C.; Zychlinsky, A. Neutrophil Extracellular Traps: How to Generate and Visualize Them. J. Vis. Exp. 2010, No. 36, 3638.

(2) Stein, F.; Kress, M.; Reither, S.; Piljić, A.; Schultz, C. FluoQ: A Tool for Rapid Analysis of Multiparameter Fluorescence Imaging Data Applied to Oscillatory Events. ACS Chem.

Biol. 2013, 8 (9), 1862-1868. 